\title{
Subspace Rejection for Matching Pursuit in Presence of Unresolved Targets
}

\author{
Jonathan Bosse and Olivier Rabaste \\ ONERA, The French Aerospace Lab BP 8010091123 Palaiseau Cedex, France
}

\begin{abstract}
Unresolved scatterers (separated by less than a 3 dB matched filter main lobe width) are known to degrade the matching pursuit performances in radar: it tends to generate spurious detection or miss weaker targets, hidden in strong sidelobes. In this article, we propose a new matching pursuit algorithm performing a subspace radar resolution cell rejection. The philosophy is the following: as it is usually not possible to distinguish several unresolved scatterers, we do not try to distinguish them, but when a scatterer is detected, then every contribution in the corresponding resolution cell should be cleaned. For that, the continuous cell interval is approximated by a subspace and the corresponding orthogonal projector is build. The projector basis is chosen so that it minimizes the projection residue inside the interval and a method is proposed to select its dimension according to the rejected target SNR. We show that it enables to control the sidelobe level after rejection (orthogonal projection), so that the sidelobe detection probability can be maintained low. It allows matching pursuit to work well even if the target parameter distribution differs from a delta Dirac distribution inside the matched filter resolution cell.
\end{abstract}

Index Terms-Unresolved targets, Clean algorithm, Matching Pursuit, Radar signal processing, Discrete Prolate Spheroidal Sequences

\section{INTRODUCTION}

$\mathbf{T}$ ARGET localization has always received great attention in the signal processing community. Such task is usually performed thanks to electromagnetic sensors (that can form a network) and the literature offers a plethora of solution to deal with this problem [1]. If several independent samples of the observation vector are available at the output of the sensor or array of sensors, then all the well known high resolution techniques (MUSIC, ESPRIT...) [1], [2] are very efficient. Unfortunately, sometimes (e.g. in radar or sonar) only a single observation is available. In this case, such techniques do not apply and achieving high resolution is quite unlikely [3].

The proposed approach is based on very general models, and can be applied in spectral estimation, sonar or more generally to array processing problems using matching pursuit (MP) [4] in presence of extended targets. Nevertheless, we will mainly focus on the radar paradigm in the sequel of the paper. In this study, we will consider that a target may be composed by one (or several) scatterer(s) with non Dirac distribution in the parameter space. As an example, it can be composed of unresolved scatterers (separated by less than the Rayleigh distance: the matched filter (MF) main lobe $3 \mathrm{~dB}$ width) or of non-Dirac Doppler spectrum scatterers (radar clutter).

Traditionally, the active radar signal processing chain consists of a (single target) MF processing followed by a detection step [5], [6] in order to both detect and locate the target. The MF is usually evaluated on a grid whose step is proportional to the resolution capability of the radar. This procedure, when performed iteratively and followed by a rejection step (orthogonal projection to the estimated contribution), can be seen as a clean [7] or equivalently matching pursuit algorithm [4], popular in compressive sensing (CS) [8]. Although CS for radar exists (see for example [9]), the radar signal is usually not "compressed", that is to say no (random) sensing matrix is applied to the signals. Consequently, we are not dealing with CS in this paper. Moreover, compressive sensing approaches face the well-known "off-grid issue" [10], and if "off the grid" algorithms have been proposed [11-16], they do not tackle unresolved targets. Strongly linked with CS, the sparse approach is attractive for the radar community as it can be seen as a smart way to handle the multiple target estimation and detection problem, whereas the corresponding maximum likelihood solution is usually unfeasible. MP is then a very good candidate as it exploits MF based estimation and detection stages which have already been intensively studied by the radar community, so that lots of results are at our disposal. For example, the MP may also easily include any well-known CFAR procedure [6] or non Gaussian detection test to deal with heterogeneous or non Gaussian clutter.

If there is more than one dominant scatterer inside a resolution cell (unresolved targets), the MF estimator can become so biased that the rejection step will perform poorly and spurious detection will be generated by the MP algorithm. These strong sidelobe residues may then prevent the detection of weaker targets. Since high resolution in radar is only possible in not too complex scenarios [3], the separation of unresolved scatterers is usually unlikely. Sometimes, due to the presence of dense clutter, the common point spread target assumption in radar is not realistic. To the best of our knowledge, the issue of unresolved targets in radar has mainly been investigated for (monopulse) Angle of Arrival estimation (see [17] and references inside). [18] proposes to oversample by two the MF grid and build a rank 2 projector, which can be computationally intensive and may not be efficient for high signal-to-noise ratio (SNR) targets as we will show.

Other efficient alternatives exist like the Capon inspired algorithm named Iterative adaptive approach (IAA), proposed in [19], or the Refined LIKelihood-based Estimation of Sparse (ReLIKES) parameters [20]. Those techniques offer high resolution (compared to the single target matched filter) possibilities, using a fine parameter grid, enabling to distinguish unresolved targets provided their location fall onto the pa- 
rameter grid. The main drawback of those approaches is the computational cost that drastically increases with the grid size. Studies like [21] have been devoted to cost reduction of the IAA approach but it only concerns a specific case of steering vector. Contrary to MF, IAA and ReLIKES are not initially designed for detection. To do so, it is sometimes associated with the Bayesian Information criterion [19], [20], but to the best of our knowledge, its statistical behavior and consequently the resulting probability of false alarm are unknown.

In this article, in order to deal with unresolved targets in active radar, we propose a new MP algorithm with an orthogonal projection (rejection) on the estimated cell subspace. The philosophy is the following: we do not try to estimate precisely the position of the targets (as we are limited by the MF resolution anyway ), but once a (possibly extended, unresolved) target has been detected, then all possible contributions belonging to the corresponding resolution cell should be removed from the measured signal to ensure a proper detection at the next iteration. For that, the cell is approximated by a subspace of appropriate dimension and then one proceeds to the corresponding orthogonal projection. It tries to remove any contribution located in the continuous interval defined by the considered cell, contrary to the classic processing where only the cell-center contribution is removed. After having introduced a general method, we will particularly focus on two widespread applications: the pulsed linear chirp waveform radar and the frequency modulated continuous waveform (FMCW) radar. The present work extends [22] where only the Doppler was investigated for passive radar, and [23] were only the case of FMCW was studied. In [23], the projection is realized thanks to the Discrete Prolate Spheroidal Sequences (DPSS) vectors [24] and the proposed processing becomes particularly simple and computationally inexpensive.

Note that DPSS vectors have recently been used for compressed sensing [25], in order to build a good compressive dictionary. Although [26] has suggested the use of DPSS for interference cancellation in compressed sensing for radio, the goal was not to clean unresolved targets. In MIMO radar, Prolate Spheroidal Wave Functions have been used to successfully model the clutter [27]. Spheroidal Sequences have also been used to improve the performance of MUSIC in [28]. In [29], DPSS are studied in order to approximate multi band signals. DPSS vectors have also been recently used for wall clutter mitigation [30], [31], but in these studies if the DPSS basis is used for orthogonal projection, the rejection is not performed according to the target SNR, contrary to what we propose in the present paper. [32] proposes to tune the dimension of the subspace, but contrary to the present work, no connection was made with the sidelobes detection probability. We show here that the choice of DPSS arises naturally and how it can be adapted to the classic active radar framework. Also, the use of DPSS vectors appears to be a particular case of the proposed method, and as we will see, this basis is no longer optimal with respect to the projection residual error when the model is different. Interestingly, although it was derived from a different approach, the subspace used in ReLIKES [20] to approximate the grid interval, share a similar structure than the one used in the present paper. But the subspace dimension is determined by a quite arbitrary criterion based on the ratio on specific eigenvalues and is independent of the target SNR, contrary to what is done in the present study.

The outline of the paper lies as follows: in the second section, we propose the radar signal model. In section III, the core of the proposed algorithm is discussed. In section IV, some widespread models are studied. Finally, in section $\mathrm{V}$, simulations show the advantages of the proposed method compared to the literature in presence of unresolved targets.

In the paper, we will adopt the following notations: $(.)^{T}$, $(.)^{*}$ and $(.)^{H}$ denote transpose, conjugate and Hermitian transpose, respectively; bold lower case letters denote vectors, whereas bold upper case letters denote matrices; $\odot$ and $\otimes$ denote the Hadamard and Kronecker products, respectively; $\theta \sim \mathcal{U}(I)$ means that the random variable $\theta$ follows a uniform distribution on the interval $I ; \mathbb{E}_{\theta}$ [.] stands for the mathematical expectation with respect to $\theta ; \lambda_{\max }(\mathbf{A})$ and $\lambda_{n}(\mathbf{A})$ denotes the maximal and the $n$-th eigenvalue of any matrix $\mathbf{A}$, respectively.

\section{SIGNAL MODEL AND PROBLEM FORMULATION}

We consider the following general model:

$$
\mathbf{y}=\sum_{m=1}^{M} \alpha_{m} \mathbf{u}\left(\boldsymbol{\theta}_{m}\right)+\mathbf{n},
$$

where $\mathbf{y}$ is an $N \times 1$ complex observation vector, $\boldsymbol{\theta}_{m}$ stands for the unknown parameter of interest, $\alpha_{i}$ is an unknown deterministic complex nuisance parameter (Swerling 0 [6] target backscattered amplitude), $\mathbf{u}($.$) is a known function and \mathbf{n}$ is an additive noise (assumed here white complex circular Gaussian with covariance matrix $\sigma^{2} \mathbf{I}$ ). We assume here for simplicity that the noise power $\sigma^{2}$ is known, but it can be easily estimated [6], independently from the parameters of interest. Without loss of generality, we assume that $\|\mathbf{u}()\|=$.1 . Such a model (1) is very general and in case of radar, $\mathbf{u}($.$) stands for the$ transmitted waveform and $\boldsymbol{\theta}$ contains for instance the range and Doppler frequency shift of target $m$. In classic radar processing, the unknown parameters $\boldsymbol{\theta}_{m}$ are estimated on a discrete set of matched filtered samples, spaced by the size of the resolution cell. Such a strategy may lead to a certain mismatch for targets located at the edge of the resolution cell. In this paper, contrary to what is usually done, we will assume a prior density on $\boldsymbol{\theta}_{m}$, in order to take the lack of knowledge on the target location within the MF resolution cell (main lobe $3 \mathrm{~dB}$ width) [3] into account. As a natural choice, we will pick the uniform distribution on the matched filter resolution cell. As we will see, this will enable us to cope with off-grid possibly unresolved - targets.

In order to estimate all parameters $\boldsymbol{\theta}_{m}$, the MP algorithm is well suited, especially if the scene is sparse, that is to say if $M \ll N$. MP can be viewed in the radar context as a clean algorithm [7]. The philosophy of this approach, summarized in Figure 1, is the following: estimate iteratively the strongest components of the signal (in the MF magnitude sense) and remove them from the original signal.

Following a classic radar detection procedure, we stop the MP procedure when the matched filter output does not exceed 


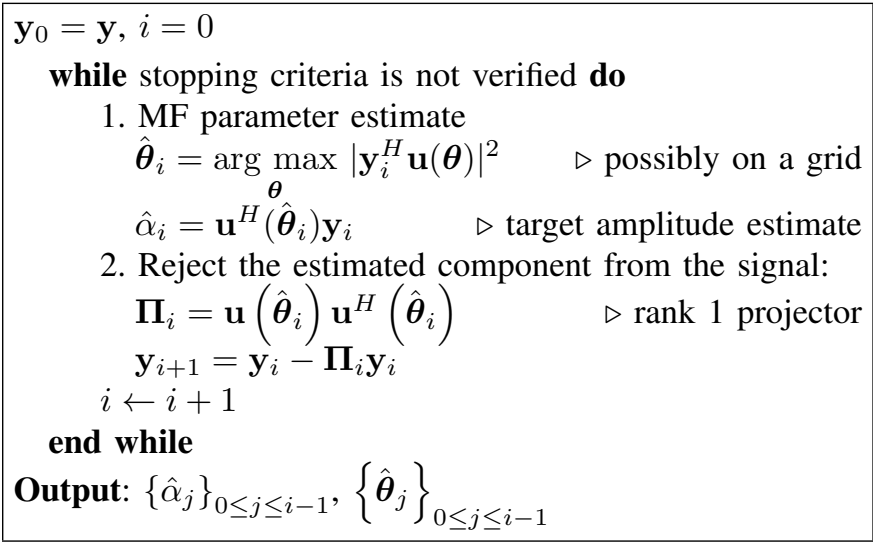

Figure 1: Matching pursuit (or clean) algorithm in radar.

a given threshold for any parameter value. This detection threshold may be set according to the single target radar detection problem, where two hypotheses are compared: the received signal $\mathbf{y}$ is either composed of the noise $\mathbf{n}$ only $\left(\mathcal{H}_{0}\right)$ or composed of the target signal $\mathbf{s}=\alpha \mathbf{u}(\boldsymbol{\theta})$ plus noise $\left(\mathcal{H}_{1}\right)$ :

$$
\begin{cases}\mathcal{H}_{0}: & \mathbf{y}=\mathbf{n} \\ \mathcal{H}_{1}: & \mathbf{y}=\alpha \mathbf{u}(\boldsymbol{\theta})+\mathbf{n}\end{cases}
$$

For a fixed parameter $\boldsymbol{\theta}$, the solution is given by the generalized likelihood ratio test (GLRT)

$$
\left|\mathbf{y}^{H} \mathbf{u}(\boldsymbol{\theta})\right|^{2} \underset{\mathcal{H}_{1}}{\stackrel{\mathcal{H}_{0}}{\lessgtr}} \gamma
$$

where the threshold $\gamma=-\sigma^{2} \log \left(P_{f a}\right)$ is set according to a desired probability of false alarm $P_{f a}$ [6].

It is known that the stopping rule influences the Matching Pursuit algorithm performance. In [33] conditions on the amplitudes of the targets and stopping rules are given so that the orthogonal matching pursuit (OMP) algorithm can offer perfect signal support recovery with high probability in the presence of Gaussian noise. Analyzing the performance of signal recovery is out of the scope of the present paper. To propose a new algorithm, we follow the simple intuition that if the rejection (or cleaning) step is properly made at each iteration, then the detection test (3) performed on the classic MF grid, will act as a reasonable stopping rule. This explains why we study in this paper the influence of the rejection stage on the following detection stage (at next iteration).

Our goal in this paper is to improve the orthogonal projection step (2) in Algorithm In presence of unresolved targets, the classic MF may fail to separate the corresponding scatterers inside the resolution cell, and the estimation step will be so biased that the rejection will perform poorly. Then, the MP tends to produce spurious detections or can even misdetect weaker targets hidden in stronger target sidelobes. Our objective in this paper is to solve these two problems using an MP based approach. Precise location estimation of one or several high SNR targets is not our primary goal: we would like to ensure that once estimated, their contribution will be properly cleaned (in other words, we would like to minimize the residues after projection) so that weaker targets possibly hidden in their sidelobes can be detected. Note that the proposed method is also useful for radar target extraction: since we aim at suppressing the strong target sidelobes, the sidelobes mitigation issue should be significantly reduced.

\section{SUBSPACE REJECTION}

In order to improve the rejection (cleaning) step of the MP algorithm, we propose to approximate the resolution cell by a subspace and then proceed to the cleaning thanks to an orthogonal projection. Note that contrary to common MP procedures, the cleaning is here performed with an other basis (or dictionary) than the one used for the detection step, where the classical MF dictionary is employed. First, we show how to choose the optimal subspace basis and then how to choose its dimension according to the target SNR. The proposed study can be easily extended to the case of a multidimensional parameter, but, for the sake of simplicity, we focus here on the scalar parameter case.

\section{A. Building the cell subspace orthogonal projection}

Recall that the orthogonal projector on any matrix $\mathbf{A}$ is:

$$
\boldsymbol{\Pi}(\mathbf{A})=\mathbf{A}\left(\mathbf{A}^{H} \mathbf{A}\right)^{\dagger} \mathbf{A}^{H},
$$

where $(.)^{\dagger}$ denotes the Moore-Penrose pseudo-inverse, which is equal to the classic inverse when the matrix is invertible. Let us also recall that, by definition, any orthogonal projector is Hermitian and idempotent (i.e. $\boldsymbol{\Pi}(\mathbf{A})=\boldsymbol{\Pi}^{H}(\mathbf{A})$ and $\left.\boldsymbol{\Pi}(\mathbf{A})^{2}=\boldsymbol{\Pi}(\mathbf{A})\right)$.

Let $\theta_{0}$ be a given sample in the MF grid and $\mathcal{R}\left(\theta_{0}, \Delta\right)=$ $\left\{\theta /\left|\theta-\theta_{0}\right|<\frac{\Delta}{2}\right\}$ be a given rejection interval of width $\Delta$, centered on $\theta_{0}$. By default, since below that limit there is very few chances that one can distinguish targets [3], $\Delta$ corresponds to the MF resolution cell width and $\theta_{0}$ belongs to the MF grid. $\Delta$ is usually such that $\mathbf{u}(\theta)$ and $\mathbf{u}(\theta+\Delta)$ are orthogonal. The domain of $\theta$ is here divided in $N$ intervals of equal size. In order to perform the continuous rejection in step 2) of the matching pursuit algorithm, we propose to consider the projector $\Pi$, that minimizes the averaged projection residue over the region $\mathcal{R}\left(\theta_{0}, \Delta\right)$ :

$$
\mathcal{E}\left(\theta_{0}, \Delta, \boldsymbol{\Pi}\right)=\mathbb{E}_{\theta}\left[\left\|\boldsymbol{\Pi}^{\perp} \mathbf{u}(\theta)\right\|^{2}\right]
$$

where we defined

$$
\Pi^{\perp}=\mathbf{I}-\mathbf{\Pi} .
$$

A prior distribution should be chosen for $\theta$. As a natural choice, we consider the uniform distribution on $\mathcal{R}\left(\theta_{0}, \Delta\right)$, although other distributions can be used. In this case, the averaged projection residue is

$$
\mathcal{E}\left(\theta_{0}, \Delta, \boldsymbol{\Pi}\right)=\frac{1}{\Delta} \int_{\theta_{0}-\frac{\Delta}{2}}^{\theta_{0}+\frac{\Delta}{2}}\left\|\boldsymbol{\Pi}^{\perp} \mathbf{u}(\theta)\right\|^{2} d \theta .
$$

The following result will allow us to construct the desired projector.

Proposition 1. Let $\Pi_{K}$ denote an orthogonal projector of rank $K$. Then if we denote $\mathbf{v}_{k}\left(\theta_{0}, \Delta\right)$ the $k^{\text {th }}$ eigenvector with 
corresponding eigenvalue $\lambda_{k}\left(\theta_{0}, \Delta\right)$ (ordered in decreasing order) of the matrix

$$
\mathbf{U}\left(\theta_{0}, \Delta\right)=\mathbb{E}_{\theta}\left[\mathbf{u}(\theta) \mathbf{u}^{H}(\theta)\right],
$$

one finds

$$
\begin{gathered}
\arg \min _{\boldsymbol{\Pi}_{K}} \mathcal{E}\left(\theta_{0}, \Delta, \boldsymbol{\Pi}_{K}\right)=\sum_{k=1}^{K} \mathbf{v}_{k}\left(\theta_{0}, \Delta\right) \mathbf{v}_{k}^{H}\left(\theta_{0}, \Delta\right), \\
\min _{\boldsymbol{\Pi}_{K}} \mathcal{E}\left(\theta_{0}, \Delta, \boldsymbol{\Pi}_{K}\right)=\sum_{k=K+1}^{N} \lambda_{k}\left(\theta_{0}, \Delta\right) .
\end{gathered}
$$

Proof: We have

$$
\begin{aligned}
\mathcal{E}\left(\boldsymbol{\theta}_{0}, \boldsymbol{\Delta}, \boldsymbol{\Pi}\right) & =\mathbb{E}_{\theta}\left[\left\|\boldsymbol{\Pi}^{\perp} \mathbf{u}(\theta)\right\|^{2}\right] \\
& =\operatorname{Tr}\left[\mathbb{E}_{\theta}\left[(\mathbf{I}-\boldsymbol{\Pi}) \mathbf{u}(\theta) \mathbf{u}^{H}(\theta)\right]\right] \\
& =\operatorname{Tr}\left[\mathbf{U}\left(\theta_{0}, \boldsymbol{\Delta}\right)\right]-\operatorname{Tr}\left[\boldsymbol{\Pi} \mathbf{U}\left(\theta_{0}, \boldsymbol{\Delta}\right)\right]
\end{aligned}
$$

and since the first term in (11) is constant with respect to $\boldsymbol{\Pi}$, the minimum of $\mathcal{E}\left(\theta_{0}, \Delta, \boldsymbol{\Pi}\right)$ is found by maximizing $\operatorname{Tr}\left[\boldsymbol{\Pi} \mathbf{U}\left(\theta_{0}, \boldsymbol{\Delta}\right)\right]$. Since $\mathbf{U}\left(\theta_{0}, \Delta\right)$ is Hermitian, we can write

$$
\mathbf{U}\left(\theta_{0}, \Delta\right)=\sum_{k=1}^{N} \lambda_{k}\left(\theta_{0}, \Delta\right) \mathbf{v}_{k}\left(\theta_{0}, \Delta\right) \mathbf{v}_{k}^{H}\left(\theta_{0}, \Delta\right) .
$$

Applying the Lemma 1 to our problem gives the desired results (9) and (10).

Lemma 1. Let $\Pi_{K}=\sum_{k=1}^{K} \boldsymbol{\pi}_{k} \boldsymbol{\pi}_{k}^{H}$ be a $N \times N$ orthogonal projector of rank $K$, then for any given $N \times N$ Hermitian matrix $\mathbf{B}=\sum_{k=1}^{N} \lambda_{k} \mathbf{v}_{k} \mathbf{v}_{k}^{H}$,

$$
\operatorname{Tr}\left[\boldsymbol{\Pi}_{K} \mathbf{B}\right] \leq \sum_{k=1}^{K} \lambda_{k},
$$

where $\lambda_{k}$ are ordered in decreasing number. The equality is achieved when $\boldsymbol{\pi}_{k}=\mathbf{v}_{k},(1 \leq k \leq K)$.

Proof: See Appendix A.

Since $\|\mathbf{u}(\theta)\|^{2}=1$, we have $\sum_{n=1}^{N} \lambda_{n}(\theta, \Delta)=1$.

Proposition 1 provides the projector that minimizes the error in the rejection area $\mathcal{R}\left(\theta_{0}, \Delta\right)$ thanks to (9) but also enables to quantify the level of rejection thanks to (10).

The study [20], based on a different approach, considered the eigenvectors of (8), with $\Delta$ being the refined grid step.

We showed in [23] that the continuous projector can also be interpreted as the limit of the orthogonal projector that would be built with several samples inside the desired interval, when the number of samples grows infinitely.

Note that when the steering vector obeys a 2D Kronecker structure, the eigenvectors are simply deduced from the original single dimension problems [23].

Note also that when the dimension of the projector $K$ increases, the residual energy within the rejection interval decreases. So, the stronger the SNR of targets within $\mathcal{R}\left(\theta_{0}, \Delta\right)$, the larger $K$ should be to ensure that the component is properly suppressed from the original signal. In the next section, we focus on the choice of this parameter depending on the SNR within $\mathcal{R}\left(\theta_{0}, \Delta\right)$.

\section{B. Choosing the dimension of the cell subspace projector}

In order to choose the dimension $K$ of the proposed continuous projector $\Pi_{K}$, a criteria that seems judicious is that the MF magnitude should be at most at the noise level in the considered cell after the rejection step. Therefore, let us consider the maximal value of the MF applied to the output of the rejection step, averaged for any target of amplitude $\alpha$, located in $\theta^{\prime}$, where $\theta^{\prime} \sim \mathcal{U}\left(\mathcal{R}\left(\theta_{0}, \Delta\right)\right)$ :

$$
\max _{\theta} \mathbb{E}_{\theta^{\prime}}\left[\left|\alpha \mathbf{u}^{H}\left(\theta^{\prime}\right) \boldsymbol{\Pi}_{K}^{\perp} \mathbf{u}(\theta)\right|^{2}\right]=|\alpha|^{2} \mathcal{C}_{\theta_{0}, \Delta}\left(\boldsymbol{\Pi}_{K}\right),
$$

where we defined

$$
\mathcal{C}_{\theta_{0}, \Delta}\left(\boldsymbol{\Pi}_{K}\right) \triangleq \max _{\theta} \mathbb{E}_{\theta^{\prime}}\left[\left|\mathbf{u}^{H}\left(\theta^{\prime}\right) \boldsymbol{\Pi}_{K}^{\perp} \mathbf{u}(\theta)\right|^{2}\right] .
$$

We propose to choose the dimension $K$ as soon as

$$
|\alpha|^{2} \mathcal{C}_{\theta_{0}, \Delta}\left(\boldsymbol{\Pi}_{K}\right) \leq \varepsilon \sigma^{2},
$$

where $\varepsilon$ is a constant typically smaller than 1 and the unknown signal to noise ratio $\frac{|\alpha|^{2}}{\sigma^{2}}$ can be estimated by $\left|\mathbf{u}^{H}\left(\theta_{0}\right) \mathbf{y}\right|^{2} / \sigma^{2}$.

Note that $\mathcal{C}_{\theta_{0}, \Delta}\left(\boldsymbol{\Pi}_{K}\right)$ may not be always easy to compute. However let us remark that [34]

$$
\begin{aligned}
\mathcal{C}_{\theta_{0}, \Delta}\left(\boldsymbol{\Pi}_{K}\right) & =\max _{\theta} \mathbf{u}^{H}(\theta) \boldsymbol{\Pi}_{K}^{\perp} \mathbf{U}\left(\theta_{0}, \Delta\right) \boldsymbol{\Pi}_{K}^{\perp} \mathbf{u}(\theta),(17) \\
& \leq \lambda_{\max }\left(\boldsymbol{\Pi}_{K}^{\perp} \mathbf{U}\left(\theta_{0}, \Delta\right) \boldsymbol{\Pi}_{K}^{\perp}\right) .
\end{aligned}
$$

If $\Pi_{K}^{\perp}$ of rank $K$ is selected according to (9), $\lambda_{\max }\left(\boldsymbol{\Pi}_{K}^{\perp} \mathbf{U}\left(\theta_{0}, \Delta\right) \boldsymbol{\Pi}_{K}^{\perp}\right)=\lambda_{K+1}\left(\theta_{0}, \Delta\right)$. Then, the rank $K$ can be selected as soon as

$$
\frac{|\alpha|^{2}}{\sigma^{2}} \lambda_{K+1}\left(\theta_{0}, \Delta\right) \leq \varepsilon
$$

Note that due to the Cauchy-Swchartz inequality

$$
\mathcal{C}_{\theta_{0}, \Delta}\left(\boldsymbol{\Pi}_{K}\right) \leq \mathbb{E}_{\theta^{\prime}}\left[\left\|\boldsymbol{\Pi}^{\perp} \mathbf{u}\left(\theta^{\prime}\right)\right\|^{2}\right]=\mathcal{E}\left(\theta_{0}, \Delta, \boldsymbol{\Pi}_{K}\right),
$$

so that if $\Pi^{\perp}$ is selected according to (9), we also have

$$
\mathcal{C}_{\theta_{0}, \Delta}\left(\boldsymbol{\Pi}_{K}\right) \leq \sum_{n=K+1}^{N} \lambda_{n}\left(\theta_{0}, \Delta\right) .
$$

As a consequence, it would also make sense to substitute $\sum_{n=K+1}^{N} \lambda_{n}\left(\theta_{0}, \Delta\right)$ to $\lambda_{K+1}\left(\theta_{0}, \Delta\right)$ in (19). Both give quite similar results when the eigenvalues are quickly decreasing, which is usually the case here.

The criteria (14) has also a more sound statistical justification: it bounds the maximum averaged (over a target in the rejection zone) probability of sidelobe detection after rejection $\bar{P}_{s}^{\max }$. Let us define the sidelobe detection probability of the matched filter at a given position $\theta$, given a target of amplitude $\alpha$ at a random position $\theta^{\prime}$ after projection

$$
P_{s}\left(\theta \mid \theta^{\prime}\right)=\operatorname{Pr}\left(\left|\left(\alpha \mathbf{u}\left(\theta^{\prime}\right)+\mathbf{n}\right)^{H} \boldsymbol{\Pi}^{\perp} \mathbf{u}(\theta)\right|^{2}>\gamma \mid \theta^{\prime}\right),
$$

where $\theta^{\prime} \sim \mathcal{U}\left(\mathcal{R}\left(\theta_{0}, \Delta\right)\right)$. Since this quantity depends on the unknown target position $\theta^{\prime}$, we define the averaged detection probability

$$
\bar{P}_{s}(\theta)=\mathbb{E}_{\theta^{\prime}}\left[P_{s}\left(\theta \mid \theta^{\prime}\right)\right] .
$$


Finally $\bar{P}_{s}^{\max }$ is defined as

$$
\bar{P}_{s}^{\max }=\max _{\theta} \bar{P}_{s}(\theta),
$$

where the maximum is taken over all possible values of $\theta$.

Proposition 2. If the projector $\Pi_{K}^{\perp}$ is selected according to (9), then

$$
\bar{P}_{s}^{\max } \leq \frac{\sigma^{2}}{\gamma}\left(1+\frac{|\alpha|^{2}}{\sigma^{2}} \lambda_{K+1}\left(\theta_{0}, \Delta\right)\right) .
$$

Proof: Applying Markov's inequality in equation (22) on the positive random variable $\epsilon=\left|\left(\alpha \mathbf{u}\left(\theta^{\prime}\right)+\mathbf{n}\right)^{H} \boldsymbol{\Pi}^{\perp} \mathbf{u}(\theta)\right|^{2}$, $\theta^{\prime}$ being fixed, we have

$$
P_{s}\left(\theta^{\prime} \mid \theta\right) \leq \frac{1}{\gamma} \mathbb{E}_{\mathbf{n}}[\epsilon],
$$

with

$$
\mathbb{E}_{\mathbf{n}}[\epsilon]=|\alpha|^{2}\left|\mathbf{u}\left(\theta^{\prime}\right)^{H} \boldsymbol{\Pi}^{\perp} \mathbf{u}(\theta)\right|^{2}+\sigma^{2}\left\|\boldsymbol{\Pi}^{\perp} \mathbf{u}(\theta)\right\|^{2} .
$$

Then, combining (23), (27) and (26), gives

$$
\begin{gathered}
\bar{P}_{s}(\theta) \leq \frac{|\alpha|^{2}}{\gamma} \mathbb{E}_{\theta^{\prime}}\left[\left|\mathbf{u}(\theta)^{H} \boldsymbol{\Pi}^{\perp} \mathbf{u}\left(\theta^{\prime}\right)\right|^{2}\right]+ \\
\frac{\sigma^{2}}{\gamma}\left\|\boldsymbol{\Pi}^{\perp} \mathbf{u}(\theta)\right\|^{2}
\end{gathered}
$$

Since

$$
\left\|\boldsymbol{\Pi}^{\perp} \mathbf{u}(\theta)\right\|^{2} \leq 1,
$$

one finds

$$
\bar{P}_{s}^{\max } \leq \frac{|\alpha|^{2}}{\gamma} \mathcal{C}_{\theta_{0}, \Delta}\left(\boldsymbol{\Pi}_{K}\right)+\frac{\sigma^{2}}{\gamma},
$$

and combining with (17) we finally have (25).

From Proposition 2, it appears quite natural to control $\bar{P}_{s}^{\text {max }}$ by selecting an appropriate dimension $K$ such that $\frac{|\alpha|^{2}}{\sigma^{2}} \lambda_{K+1}\left(\theta_{0}, \Delta\right)$ remains small for a given target SNR, or, in other words, to increase the rank $K$ when the SNR grows. Choosing $\lambda_{K+1}$ such that $\frac{|\alpha|^{2}}{\sigma^{2}} \lambda_{K+1} \ll 1, \bar{P}_{s}^{\max }$ is then approximately bounded by $\frac{\sigma^{2}}{\gamma}=\frac{-1}{\log \left(P_{f a}\right)}$. This explains why it is interesting to select the rank of the projector according to the target SNR. Selecting the rank of the projector for the SNR measured in the considered cell allows us to control the probability of false alarm after rejection, and selecting the projection basis according to (9) provides the fastest decay of the eigenvalues. This nice feature is not shared by existing algorithms: with the classic rank-one projector, then $\bar{P}_{s}^{\max }$ may reach unacceptable values depending on the target SNR. This is why a classic MP approach based on simple rank-one projectors is not suited to unresolved targets.

Unfortunately, the bound (25) is not very tight: for instance, for a typical radar application with $P_{f a} \approx 10^{-4}$, the bound on $\bar{P}_{s}^{\text {max }}$ is about 0.1 . However in reality, when $\Pi_{K}^{\perp}$ is selected according to (9) and (19), the false alarm probability $\bar{P}_{s}^{\max }$ is often much less. Ideally it should be close to $P_{f a}$.In order to obtain a tighter bound, we will introduce some approximations. As we shall see in the sequel, these approximations are quite realistic for the considered case.
Proposition 3. If

$$
\mu=\max _{\theta \in \mathcal{R}\left(\theta_{0}, \Delta\right)}\left\|\boldsymbol{\Pi}^{\perp} \mathbf{u}(\theta)\right\|^{2}
$$

is such that $\frac{|\alpha|^{2}}{\sigma^{2}} \mu<-\log \left(P_{f a}\right)$,

$$
\bar{P}_{s}^{\max } \leq e^{-\left(\sqrt{\frac{\gamma}{\sigma^{2}}}-\sqrt{\frac{|\alpha|^{2}}{\sigma^{2}} \mu}\right)^{2}} .
$$

Moreover, if $\frac{|\alpha|^{2}}{\sigma^{2}} \mu$ tends to zero then

$$
\bar{P}_{s}^{\text {max }} \leq P_{f a}\left(1+\mathcal{O}\left(\sqrt{\frac{|\alpha|^{2}}{\sigma^{2}} \mu}\right)\right) .
$$

Proof: See Appendix D.

Unfortunately, in general, we do not have much information on $\mu$ but some approximations may be useful. First, we will assume that when $K$ is sufficiently large, if $\Pi^{\perp}$ is chosen according to (9), then $\left\|\boldsymbol{\Pi}_{K}^{\perp} \mathbf{u}\left(\theta^{\prime}\right)\right\|^{2}$ is assumed approximately constant for all $\theta^{\prime} \in \mathcal{R}\left(\theta_{0}, \Delta\right)$, and equal to its mean value:

$$
\begin{aligned}
\mu & \approx \mathbb{E}_{\theta^{\prime}}\left[\left\|\boldsymbol{\Pi}_{K}^{\perp} \mathbf{u}\left(\theta^{\prime}\right)\right\|^{2}\right] \\
& =\sum_{k=K+1}^{N} \lambda_{k} .
\end{aligned}
$$

Second, we assume that the $\lambda_{k}$ are quickly decreasing so that

$$
\sum_{k=K+1}^{N} \lambda_{k}\left(\theta_{0}, \Delta\right) \approx \lambda_{K+1}\left(\theta_{0}, \Delta\right) \approx \mu .
$$

Under these assumptions, it becomes possible to formulate the following approximated upper bound:

$$
\bar{P}_{s}^{m a x} \lesssim P_{f a}\left(1+\mathcal{O}\left(\sqrt{\frac{|\alpha|^{2}}{\sigma^{2}} \lambda_{K+1}\left(\theta_{0}, \Delta\right)}\right)\right) .
$$

So, it is interesting to see that when $\frac{|\alpha|^{2}}{\sigma^{2}} \lambda_{K+1}\left(\theta_{0}, \Delta\right) \ll 1$, $\bar{P}_{s}^{\text {max }}$ should be approximately quite close to $P_{f a}$. Looking more carefully at (80) $\frac{|\alpha|^{2}}{\sigma^{2}} \lambda_{K+1}\left(\theta_{0}, \Delta\right) \ll \frac{\sigma^{2}}{4 \gamma}=\frac{-1}{4 \log P_{f a}}$ seems a more reasonable choice.

One is tempted to choose a large value of $K$ so that the sidelobe detection probability becomes very low. Unfortunately, as we will see below, increasing $K$ tends to decrease the detection probability for targets located outside the rejection region. Indeed let us define for any target of amplitude $\alpha$, located in $\theta$ nearby $\mathcal{R}\left(\theta_{0}, \Delta\right)$, the corresponding detection probability

$$
P_{d}(\theta)=\operatorname{Pr}\left(\left|(\alpha \mathbf{u}(\theta)+\mathbf{n})^{H} \boldsymbol{\Pi}^{\perp} \mathbf{u}(\theta)\right|^{2}>\gamma\right) .
$$

Then we have, following the same reasoning as in (71)

$$
P_{d}(\theta)=Q_{1}\left(\frac{\sqrt{2}|\alpha|}{\sigma}\left\|\boldsymbol{\Pi}^{\perp} \mathbf{u}(\theta)\right\|, \frac{\sqrt{2 \gamma}}{\sigma\left\|\boldsymbol{\Pi}^{\perp} \mathbf{u}(\theta)\right\|}\right),
$$

where $Q_{1}(.,$.$) is defined in equation (72). When \theta$ is close to the rejection region, $\left\|\Pi^{\perp} \mathbf{u}(\theta)\right\|$ decreases and the first argument of $Q(.,$.$) in (72) decreases while the second argument$ increases so that $P_{d}(\theta)$ is decreasing. 


$$
\begin{aligned}
& \mathbf{y}_{0}=\mathbf{y}, i=0, \gamma=-\sigma^{2} \log \left(P_{f a}\right) \\
& \text { while } \max _{\theta \in \mathrm{MF} \text { grid }}\left|\mathbf{y}_{i}^{H} \mathbf{u}(\theta)\right|^{2}<\gamma \text { do } \\
& \text { 1. Matched filter: } \\
& \hat{\theta}_{i}=\arg \max \left|\mathbf{y}_{i}^{H} \mathbf{u}(\theta)\right|^{2} \\
& \hat{\alpha}_{i}=\mathbf{u}^{H}\left(\hat{\theta}_{i}\right) \mathbf{y}_{i} \quad \triangleright \text { deduce the SNR estimate } \\
& \text { 2. Subspace rejection: } \\
& \text { get }\left\{\mathbf{v}_{k}\left(\hat{\theta}_{i}, \Delta\right), \lambda_{k}\left(\hat{\theta}_{i}, \Delta\right)\right\}_{1 \leq k \leq N} \text { from } \mathbf{U}\left(\hat{\theta}_{i}, \Delta\right)^{a} \\
& \left\{\begin{array}{l}
K=\underset{k}{\arg \max } \lambda_{k}\left(\hat{\theta}_{i}, \Delta\right) \\
\text { subject to } \frac{\left|\hat{\alpha}_{i}\right|^{2}}{\sigma^{2}} \lambda_{k}\left(\hat{\theta}_{i}, \Delta\right) \leq \varepsilon
\end{array} \quad \triangleright\right. \text { rank estimate } \\
& \text { projector } \\
& \mathbf{\Pi}_{i}=\sum_{k=1}^{K} \mathbf{v}_{k}\left(\hat{\theta}_{i}, \Delta\right) \mathbf{v}_{k}^{H}\left(\hat{\theta}_{i}, \Delta\right) \\
& \triangleright \text { Subspace } \\
& \mathbf{y}_{i+1}=\mathbf{y}_{i}-\mathbf{\Pi}_{i} \mathbf{y}_{i} \\
& i \leftarrow i+1 \\
& \text { Output: }\left\{\hat{\alpha}_{j}\right\}_{0 \leq j \leq i-1},\left\{\hat{\theta}_{j}\right\}_{0 \leq j \leq i-1}
\end{aligned}
$$

${ }^{a}$ All information needed can often be stored off-line so that the singular value decomposition of the matrix is not required (see Section IV).

Figure 2: Proposed subspace rejection matching pursuit algorithm.

The proposed rejection criteria is well suited for one single off-grid scatterer located randomly in the rejection region. It is shown in Appendix $\mathrm{E}$ that our approach can be readily extended to the case of multiple unresolved scatterers, provided that the scatterers in $\mathcal{R}\left(\theta_{0}, \Delta\right)$ have quite similar phases, which we can roughly expect if the resolution cell is narrow enough. On the contrary, if the scatterers have very chaotic phases, the estimated SNR in $\mathcal{R}\left(\theta_{0}, \Delta\right)$ can be so poor that the choice of the rank of the projector in (19) may be inappropriate and the proposed rejection may perform poorly.

Note that the rejection criterion (19) proposed in this paper offers some guarantee for a good control of the probability of false alarm after rejection, at least on average, since the scatterer positions are unknown. Of course, there may exist some particular realizations where the control of the false alarm probability after rejection is not achieved.

The proposed subspace rejection matching pursuit (SR MP) algorithm is summarized in Figure 2 and can be readily adapted to the case where $\theta$ is a vector of parameters.

When the steering vector $\mathbf{u}(\theta)$ has a $2 \mathrm{D}$ Kronecker structure, the problem is separable with respect to the two dimensions and it is convenient to use the following Proposition to compute $\lambda_{\max }\left(\boldsymbol{\Pi}_{K}^{\perp} \mathbf{U}\left(\theta_{0}, \Delta\right) \boldsymbol{\Pi}_{K}^{\perp}\right)$.

Proposition 4. If $\boldsymbol{\theta}=\left[\theta_{1}, \theta_{2}\right]^{T}$ is a $2 \times 1$ vector such that

$$
\mathbf{u}(\boldsymbol{\theta})=\mathbf{u}_{1}\left(\theta_{1}\right) \otimes \mathbf{u}_{2}\left(\theta_{2}\right)
$$

with $\mathbf{u}_{1}(.) \in \mathbb{C}^{N_{1}}, \mathbf{u}_{2}(.) \in \mathbb{C}^{N_{2}}$, where $\theta_{i} \sim$ $\mathcal{U}\left(\mathcal{R}\left(\theta_{0, i}, \Delta_{i}\right)\right), i \in\{1,2\}$ are independent random variables. If $\boldsymbol{\Pi}_{K}=\boldsymbol{\Pi}_{K_{1}} \otimes \boldsymbol{\Pi}_{K_{2}}$, with $K=K_{1} K_{2}$ and $\boldsymbol{\Pi}_{K_{i}}=\sum_{n=1}^{K_{i}} \mathbf{v}_{n, i} \mathbf{v}_{n, i}^{H}, i \in\{1,2\}$ where $\mathbf{v}_{n, i}$ is the $n$-th eigenvector of $\mathbf{U}_{i}=\mathbb{E}_{\theta_{i}}\left[\mathbf{u}_{i}\left(\theta_{i}\right) \mathbf{u}_{i}^{H}\left(\theta_{i}\right)\right]$,with corresponding eigenvalue $\lambda_{n, i}\left(1 \leq n \leq N_{i}\right)$, then,

$$
\lambda_{\max }\left(\boldsymbol{\Pi}_{K}^{\perp} \mathbf{U} \boldsymbol{\Pi}_{K}^{\perp}\right)=\max \left(\lambda_{K_{1}+1,1} \lambda_{1,2}, \lambda_{1,1} \lambda_{K_{2}+1,2}\right),
$$

with $\mathbf{U}=\mathbb{E}_{\boldsymbol{\theta}}\left[\mathbf{u}(\boldsymbol{\theta}) \mathbf{u}^{H}(\boldsymbol{\theta})\right]=\mathbf{U}_{1} \otimes \mathbf{U}_{2}$.

Proof: See Appendix B.

The dimension $K$ is then selected according to the algorithm presented in Appendix C.

In this paper, we consider that the rejection region $\mathcal{R}\left(\theta_{0}, \Delta\right)$ represents the matched filter resolution cell, but it is possible, if a given target extends to several cells, to merge them all and design a new rejection interval accordingly. For the sake of brevity, this case will not be investigated in this paper.

\section{Setting the threshold $\gamma$ and alternative stopping rule}

After one MP iteration, the signal becomes $\mathbf{y}_{1}=\boldsymbol{\Pi}_{0}^{\perp} \mathbf{y}$. Due to the rejection, the probability of false alarm is lower than the initial one if the threshold $\gamma=-\sigma^{2} \log \left(P_{f a}\right)$ remains unchanged. This is not a real issue for most of the cases, but for targets that are located close to $\mathcal{R}\left(\theta_{0}, \Delta\right)$, it may be relevant to lower the threshold so that these targets can be better detected. In order to do so, one can choose as a new threshold $\gamma^{\prime}=\gamma\left\|\boldsymbol{\Pi}_{0}^{\perp} \mathbf{u}(\theta)\right\|^{2}$ so that the new detection problem is now, replacing $\mathbf{y}$ by $\mathbf{y}_{1}$ and $\gamma$ by $\gamma^{\prime}$ in (3)

$$
\frac{\left|\mathbf{y}^{H} \boldsymbol{\Pi}_{0}^{\perp} \mathbf{u}(\theta)\right|^{2}}{\left\|\boldsymbol{\Pi}_{0}^{\perp} \mathbf{u}(\theta)\right\|^{2}} \underset{\mathcal{H}_{1}}{\stackrel{\mathcal{H}_{0}}{\lessgtr}} \gamma .
$$

If we write $\boldsymbol{\Pi}_{0}^{\perp}=\mathbf{I}-\mathbf{U}_{0} \mathbf{U}_{0}^{H}$, this is the GLRT solution of the following detection problem

$$
\begin{cases}\mathcal{H}_{0}: & \mathbf{y}=\mathbf{U}_{0} \boldsymbol{\beta}+\mathbf{n} \\ \mathcal{H}_{1}: & \mathbf{y}=\alpha \mathbf{u}(\theta)+\mathbf{U}_{0} \boldsymbol{\beta}+\mathbf{n},\end{cases}
$$

where $\boldsymbol{\beta}$ is an unknown deterministic vector [35]. This test aims at detecting a target in presence of an interference term $\mathrm{U}_{0} \boldsymbol{\beta}$. The function in the left hand side of (42) is also the cost function to be maximized if one is looking for the ML estimate of $\theta$ when $\mathbf{y}=\alpha \mathbf{u}(\theta)+\mathbf{U}_{0} \boldsymbol{\beta}+\mathbf{n}$ [35]. So, both from an estimation and detection point of view, (42) is more interesting than the classic matched filter. But as a drawback, when the number of iterations $i$ increases, $\Pi_{0}^{\perp}$ in (42) should be replaced by $\boldsymbol{P}_{i-1}^{\perp}=\prod_{m=0}^{i-1} \boldsymbol{\Pi}_{m}^{\perp}$ and this increases the computational cost too. A better solution then (from a performance point of view) would be to use an orthogonal matching pursuit (OMP) approach [36] with a subspace rejection, which can be readily derived from the proposed algorithm, again with a stronger computational cost. Note that the difference between all those algorithms becomes only visible when the targets are really close to each other (very few resolution cells away).

\section{SOME WIDESPREAD MODELS}

\section{A. Sinusoids in noise}

We now study the case of a steering vector composed of pure complex phase shifts, depending on the frequency $f$

$$
\mathbf{u}(\theta)=\mathbf{a}_{P}(f)=\frac{1}{\sqrt{P}}\left[\begin{array}{lll}
1 & \ldots & e^{j 2 \pi f(P-1)}
\end{array}\right]^{T} .
$$




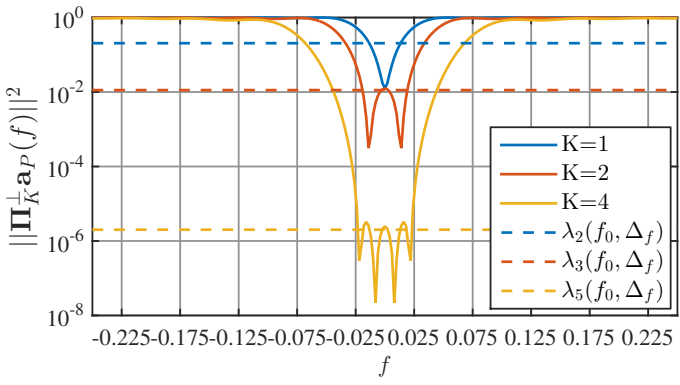

(a) $f_{0}=0, P=20, \Delta_{f}=\frac{1}{P}$

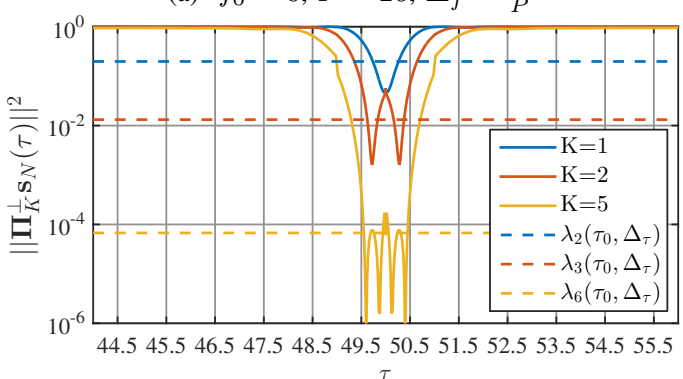

(b) $\tau_{0}=50, N=100, N_{p}=20, T_{s}=1 / B, \Delta_{\tau}=1 . \tau$ and

$\Delta_{\tau}$ are normalized by the sampling period $T_{s}=1 / B$.

Figure 3: Residual error after projection for the steering vector (44) and (49) in Figures 3a and 3b, respectively, where $\Pi$ is chosen as the optimal projector.

In this paper we will choose $\Delta_{f}=\frac{1}{P}$. In that case the matrix that has to be diagonalized is

$$
\begin{aligned}
\mathbf{A}\left(f_{0}, \Delta_{f}\right) & =\mathbb{E}_{f}\left[\mathbf{a}_{P}(f) \mathbf{a}_{P}^{H}(f)\right] \\
& =\frac{1}{\Delta_{f}} \int_{f_{0}-\frac{\Delta_{f}}{2}}^{f_{0}+\frac{\Delta_{f}}{2}} \mathbf{a}_{P}(f) \mathbf{a}_{P}^{H}(f) d f \\
& =\frac{1}{\Delta_{f}}\left[\mathbf{a}_{P}\left(f_{0}\right) \mathbf{a}_{P}^{H}\left(f_{0}\right)\right] \odot \mathbf{B}_{P, \frac{\Delta_{f}}{2}},
\end{aligned}
$$

where the matrix $\mathbf{B}_{P, W}$ is defined by

$$
\left[\mathbf{B}_{P, W}\right]_{k l}=2 W \operatorname{sinc}(2 W(k-l)), 1 \leq k, l \leq P,
$$

and where we denote $\mathbf{u}_{k}(P, W)$ and $\lambda_{k}^{\prime}(P, W)$ the corresponding $\mathrm{k}$-th eigenvector and eigenvalue respectively (sorted by decreasing magnitude).

Then, all the essential information is embedded in the eigenvectors of $\mathbf{B}_{P, W}$ which correspond to the well-known Discrete Prolate Spheroidal Sequences (DPSS) vectors [24]. These DPSS possess many interesting mathematical properties which have been extensively studied in [24]. One of their interesting feature is that the eigenvalues $\lambda_{k}^{\prime}(P, W)$ enjoy usually a very quick decay toward zero. It has been shown that their decay is exponential toward zero when $k$ grows and only a few $(2 P W)$ eigenvalues cluster to one [24]. It means that only few eigenvectors are needed for our continuous projector to achieve a good rejection. The index-limited sequence DPSS $\left\{\mathbf{u}_{k}(P, W)\right\}$ is by construction the index-limited sequence with most concentrated spectrum in $[-W, W]$ and orthogonal to $\left\{\mathbf{u}_{k}(P, W), k \in[1, n-1]\right\}, \lambda_{k}^{\prime}(P, W)$ denoting its fraction of energy in $[-W, W]$ [24]. So the result (45) is not that surprising. Also, it explains why very few eigenvectors are needed to describe the rejection interval with a high degree of accuracy.

Equation (45) can be straightforwardly rewritten as in equation (12) [23] with $\lambda_{k}\left(f_{0}, \Delta_{f}\right)=\lambda_{k}^{\prime}\left(P, \frac{1}{2 P}\right)$ and $\mathbf{v}_{k}\left(f_{0}, \Delta_{f}\right)=\mathbf{a}_{P}\left(f_{0}\right) \odot \mathbf{u}_{k}\left(P, \frac{1}{2 P}\right)$. It means that the eigenvalues and eigenvectors of $\mathbf{A}\left(f_{0}, \Delta_{f}\right)$ are readily obtained from those of $\mathbf{B}_{P, \frac{\Delta_{f}}{2}}$. These eigenvalues can be stored off-line and consequently no SVD is required which is very interesting from a computational cost point of view.

Note also that when $\Pi_{K}$ is taken according to (9)

$$
\begin{aligned}
\left\|\boldsymbol{\Pi}_{K} \mathbf{a}_{P}(f)\right\|^{2} & =\sum_{k=1}^{K} \frac{1}{P}\left|\sum_{p=1}^{P} u_{k, p}\left(P, \frac{\Delta_{f}}{2}\right) e^{j 2 \pi(p-1) f}\right|^{2} \\
& =\frac{1}{P} \sum_{k=1}^{K}\left|U_{k}\left(P, \frac{\Delta_{f}}{2} ; f\right)\right|^{2}
\end{aligned}
$$

where $U_{k}(P, W ; f)$ is by definition the $k$-th Discrete Prolate Spheroidal Wave Function (DPSWF) [24]. Consequently, with the model (44), we have an analytical expression for (31):

$$
\mu=1-\min _{f \in \mathcal{R}\left(f_{0}, \Delta_{f}\right)} \frac{1}{P} \sum_{k=1}^{K}\left|U_{k}\left(P, \frac{\Delta_{f}}{2} ; f\right)\right|^{2} .
$$

As we can see on Figure 3a, when the rank of the projector $K$ grows, the approximation (34) is quite reasonable.

\section{B. Chirp signals}

The linearly frequency modulated waveform (chirp pulse) of duration $T_{p}$, that depends on the delay $\tau$ can be written

$$
[\mathbf{u}(\theta)]_{n}=\left[\mathbf{s}_{N}(\tau)\right]_{n}=\frac{1}{\sqrt{N_{p}}} e^{j \alpha\left(t_{n}-\tau\right)^{2}} \Pi_{T_{p}}\left(t_{n}-\tau\right),
$$

where we are interested in estimating the delay $\tau$, with

$$
\Pi_{T}(t)= \begin{cases}1 & \text { if } 0 \leq t<T \\ 0 & \text { else }\end{cases}
$$

$\alpha=\pi \frac{B}{T_{p}}$ being the chirp-rate, $B$ the desired signal bandwidth and $T_{p}=N_{p} T_{s}$ the pulse period, $T_{s}$ being the sampling period. Throughout the paper, one delay resolution cell is of size $\Delta_{\tau}=1 / B$.

The matrix that has to be diagonalized is now

$$
\begin{aligned}
\mathbf{S}\left(\tau_{0}, \Delta\right) & =\mathbb{E}_{\tau}\left[\mathbf{s}_{N}(\tau) \mathbf{s}_{N}^{H}(\tau)\right] \\
& =\frac{1}{\Delta_{\tau}} \int_{\tau_{0}-\frac{\Delta_{\tau}}{2}}^{\tau_{0}+\frac{\Delta_{\tau}}{2}} \mathbf{s}_{N}(\tau) \mathbf{s}_{N}^{H}(\tau) d \tau,
\end{aligned}
$$

that can also be written in closed form,

$$
[\mathbf{S}]_{k l}= \begin{cases}\frac{2 \delta_{k l}}{\Delta_{\tau}} a_{k l}\left(\bar{\delta}_{k l}\right) \operatorname{sinc}\left(\frac{2 \alpha}{\pi} \delta_{k l}\left(t_{k}-t_{l}\right)\right) & \text { if }(k, l) \in \mathcal{U} \\ 0 & \text { else }\end{cases}
$$

with

$$
\begin{gathered}
\mathcal{U}=\left\{(k, l) / \tau_{0}-\frac{\Delta_{\tau}}{2} \leq t_{k} \leq T+\tau_{0}+\frac{\Delta_{\tau}}{2},\left|t_{k}-t_{l}\right| \leq T\right\} \\
a_{k l}\left(\bar{\delta}_{k l}\right)=\frac{1}{N_{p}} e^{j \alpha\left(t_{k}-\bar{\delta}_{k l}\right)^{2}} e^{-j \alpha\left(t_{l}-\bar{\delta}_{k l}\right)^{2}}
\end{gathered}
$$


where $\delta_{k l}=\frac{1}{2}\left(d_{k l}^{+}-d_{k l}^{-}\right)$, and $\bar{\delta}_{k l}=\frac{1}{2}\left(d_{k l}^{+}+d_{k l}^{-}\right)$, with $d_{k l}^{+}=$ $\min \left(\tau_{0}+\frac{\Delta_{\tau}^{2}}{2}, t_{k}, t_{l}\right), d_{k l}^{-}=\max \left(\tau_{0}-\frac{\Delta_{\tau}}{2}, t_{k}-T, t_{l}-T\right)$. The structure of matrix (53) is quite similar to the one in (45). Note that if $F_{s}=B$ and $\Delta_{\tau}=T_{s}$ then with $\tau_{0}=k_{0} T_{s}$

$$
\mathbf{S}\left(\tau_{0}+\frac{\Delta_{\tau}}{2}, \Delta_{\tau}\right)=\frac{1}{\Delta_{\tau}}\left[\mathbf{s}_{N}\left(\tau_{0}\right) \mathbf{s}_{N}^{H}\left(\tau_{0}\right)\right] \odot \mathbf{D}
$$

where

$\mathbf{D}_{n, m}=\left\{\begin{array}{ll}{\left[\mathbf{B}_{N_{p}, \frac{1}{2 N_{p}}}\right]_{n-k_{0}, m-k_{0}}} & \text { if } k_{0}<m, n \leq k_{0}+N_{p} \\ 0 & \text { elsewhere }\end{array}\right.$.

We see that the matrix in (56) is sparse and the non-zeros eigenvalues are those corresponding to the DPSS matrix $\mathbf{B}_{N_{p}, \frac{1}{2 N_{p}}}$. It suggests that, in general, we might expect that the eigenvalues of $\mathbf{S}\left(\tau_{0}, \Delta\right)$ are also quickly decreasing, and that the approximation (34) is quite reasonable, which is confirmed by the simulation done in Figure $3 b$.

It is interesting to note that, as in (45), the eigenvalues of the matrix (52) do not depend on the position on the grid, so that the eigenvalues needed for the rejection are those of $\mathbf{S}(0, \Delta)$. The eigenvectors of $\mathbf{S}(\tau, \Delta)$ are also easily deduced from those of $\mathbf{S}(0, \Delta)$ by an appropriate time shift. This gives the possibility to store most of the information needed for the rejection off-line, so that the computational cost is greatly reduced. Indeed, only the eigenvectors and eigenvalues of $\mathbf{S}\left(0, \Delta_{\tau}\right)$ or $\mathbf{A}\left(0, \Delta_{f}\right)$ are required, in order to build the projection basis and to select the rank $K$, respectively.

\section{Radar case (FMCW and pulsed chirp radar)}

In general, for the signal models encountered in radar, the Doppler is decoupled from the range information so that the steering vector model obeys a Kronecker structure, where the range information is carried by the waveform $s(t)$ and the Doppler is always a pure phase as in (44).

1) The case of FMCW : The FMCW radar model is [23]

$$
\begin{gathered}
\mathbf{u}(\tau, \nu)=\mathbf{a}_{P}(f(\nu)) \otimes \mathbf{a}_{N}(f(\tau)), \\
f(\nu)=\nu T_{p}, \\
f(\tau)=-\frac{\alpha}{\pi} \tau T_{s},
\end{gathered}
$$

where $T_{s}$ and $T_{p}$ are the sampling and the pulse repetition period, respectively. We also defined $\nu=2 v / \lambda$ as the unknown Doppler shift, where $\lambda$ is the carrier wavelength. The proposed algorithm has already been studied in [23]. Note that this type of radar is particularly attractive for low cost device and that the proposed algorithm is well suited, as the computational requirement is rather weak.

2) The case of pulsed chirp radar waveform: The case of pulsed chirp radar is given by the following model

$$
\mathbf{u}(\tau, \nu)=\mathbf{a}_{P}(f(\nu)) \otimes \mathbf{s}_{N}(\tau),
$$

where $P$ denotes here the number of pulses. As discussed in subsections IV-A and IV-B, taking advantage of the 2D Kronecker structure of the model (61), the proposed processing becomes very simple.

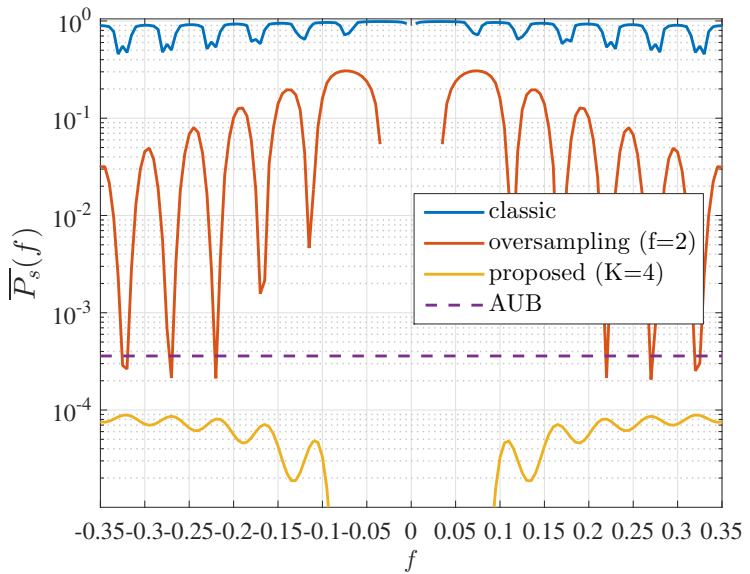

Figure 4: $\bar{P}_{s}(f)$ for the steering vector (44), $P=20, f_{0}=0$, $\Delta_{f}=\frac{1}{P}, S N R=50 \mathrm{~dB}, K=4, P_{f a}=10^{-4}$. AUB is the approximated upper bound obtained combining (77) and (36).

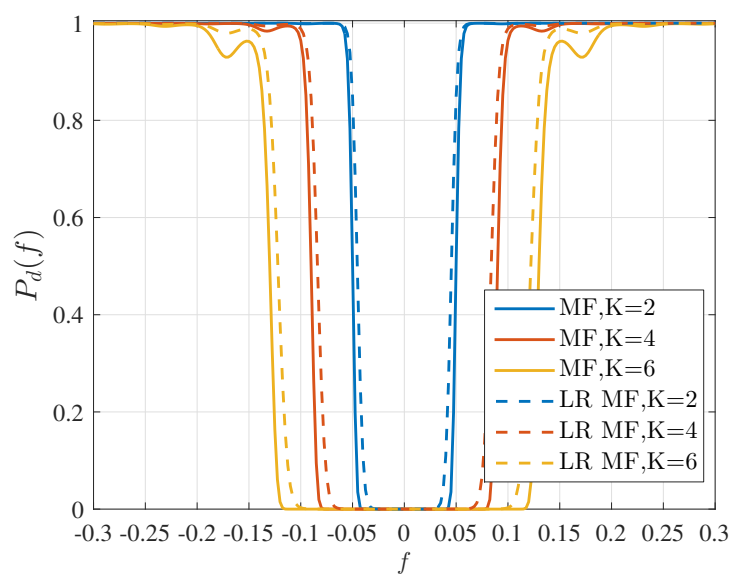

Figure 5: $P_{d}(f)$ defined in (39) for the steering vector (44), $P=20, f_{0}=0, \Delta_{f}=\frac{1}{P}, \omega_{0}=0, S N R=15 \mathrm{~dB}, P_{f a}=$ $10^{-4}$.

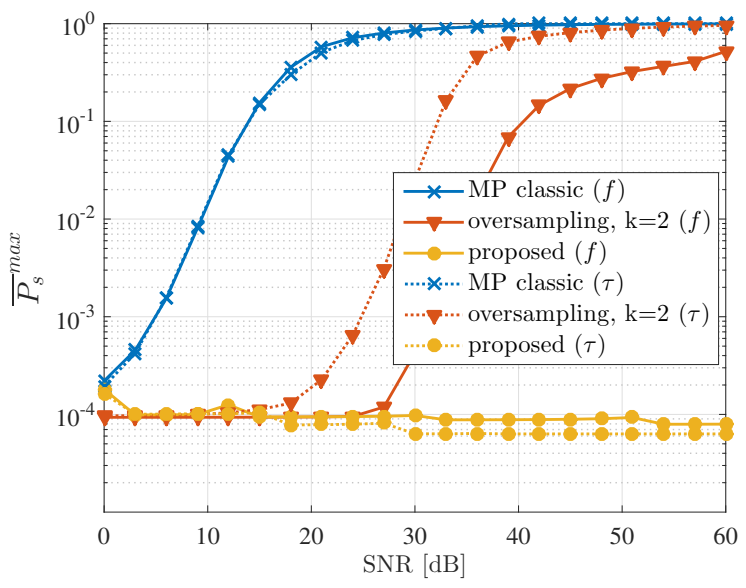

Figure 6: Maximum probability of sidelobes appearance $\bar{P}_{s}^{\max }, N=100, N_{p}=20, P=20, \Delta_{f}=\frac{1}{P}, f_{0}=0$, $\Delta_{\tau}=1 / B, \tau_{0}=N / 2, T_{s}=1 / B, P_{f a}=10^{-4}, \varepsilon=0.3$. 


\section{NUMERICAL RESULTS}

The "natural" step size of the grid in our case, is of size $1 / B$ for the delay estimation in (49) and $1 / P$ in the frequency estimation problem (44). In the sequel when we refer to finer grids, an oversampling factor $\mathrm{k}$ will be introduced, meaning that the natural step has been divided by $\mathrm{k}$.

In Figure 4, we compare the probability $\bar{P}_{s}(f)$ of detecting a sidelobe outside the region $\mathcal{R}\left(\omega_{0}, \Delta\right)$ after a rejection has been performed in this region. As expected, we can see that the classic rank one rejection is not efficient at all, the technique based on a rank 2 projector is not efficient enough as we can observe that $\bar{P}_{s}(f)$ gets close to 0.2 in the vicinity of $\mathcal{R}\left(\omega_{0}, \Delta\right)$. On the contrary when applying the proposed technique, a rank $K=4$ is provided by the criteria discussed in section III-B , and the resulting detection probability is close to the desired $P_{f a}$ (in fact less than $P_{f a}$ ). Numerical results showed us that the AUB exhibits a good agreement with $\bar{P}_{s}^{\text {max }}$ and could be used also as a rank selection criterion.

Of course, choosing a large dimension for the projector is not harmless: it tends to degrade the detection probability in the vicinity of $\mathcal{R}\left(\omega_{0}, \Delta\right)$ as we can see in Figure 5. Choosing a larger $K$ induces a performance loss that extends to a larger region outside $\mathcal{R}\left(\omega_{0}, \Delta\right)$. This is why $K$ should be as small as possible provided the rejection is sufficient. The Low Rank matched filter detector (LR MF) in equation (42) offers a slight performance improvement compared to the classic matched filter; the gain is only visible for targets close to $\mathcal{R}\left(\omega_{0}, \Delta\right)$.

In Figure 6, we compare the maximal value of the false alarm $\bar{P}_{s}^{\max }$ with respect to the rejected target SNR. The rank of the projector is selected according to the rule (19). We see that, contrary to a classic MP method (with a rank equal to one) or a technique such as [18] where the projector is generated based on an oversampled grid of factor 2, we are able to obtain a reasonable control of $\bar{P}_{s}^{\max }$ when the target SNR grows. As observed in [18], the oversampling (by a factor 2 ) is a reasonable technique when the target SNR is below 20 $\mathrm{dB}$ but when the target SNR grows it is not a relevant option. We can also observe that for the proposed method, when the SNR grows, $\bar{P}_{s}^{\max }$ is degraded until the criteria decides to choose a higher value for $K$. According to equation (80), as already discussed, choosing $\varepsilon=\frac{-1}{4 \log \left(P_{f a}\right)}$ is a reasonable choice but we found empirically that $\varepsilon=0.3$ in (19) provides good results, as confirmed by this Figure.

We now compare the following algorithms for the pulsed chirp radar waveform case (61):

- the off-grid MP.

- the OMP (off the grid).

- the MP algorithm using a rank 2 projector based on a refined $(\mathrm{k}=2)$ matched filter grid, as in [18].

- the proposed subspace rejection matching pursuit (SR MP) technique as summarized in Figure 2, with $\varepsilon=0.3$.

- the IAA, on two grids $\mathrm{k}=3$ and $\mathrm{k}=4$. To deal with off-grid estimation and detection, one applies the IAAAPES\&RELAX algorithm [19] instead, abbreviated as IAA throughout the paper. The number of iterations was set to 15 , as in [19].
- The algorithm ReLIKES [20], applied on two grids: with $\mathrm{k}=3$ and $\mathrm{k}=4$ as oversampling factor of the grid.

Note that IAA requires to iteratively inverse a grid sized matrix (in the range-Doppler estimation its dimension is then $\left.\mathrm{k}^{2} P N B T_{s}\right)$. ReLIKES is also an iterative algorithm, that requires at each step to solve a second order cone program which size is proportional to the grid size multiplied by the subspace dimension that approximates the grid interval [20]. It can be seen that the computational complexity quickly grows with the size of the grid. It is suggested in [20] to run ReLIKES with several grids and select the one that provides the weakest least squares residual. We found this approach unrealistic from a computational point of view even for the modest radar problem considered in Figure 7 (whereas in common radar problems both $N$ and $P$ can reach several hundreds) at least for current reasonably powerful computers. This is why we applied IAA and ReLIKES with grids that either contain $(\mathrm{k}=4)$ or not $(\mathrm{k}=3)$ the strongest unresolved target. The choice for $\mathrm{k}=4$ can be seen as near optimal and quite favorable, since in this dictionary the strong target is exactly sparse, but in practice, the target parameters are unknown.

The proposed method involves only basic matrix and vectors multiplications. No refined grid is required and the SVD at each iteration can usually be done off-line so that the subspace construction is particularly simple.

As we can see in Figure 7, when a target is unresolved (made of 4 scatterers here), the off-grid MP algorithm produces a lot of spurious detections: this algorithm cannot separate all contributions and performs thus poorly. The OMP technique is also not efficient and neither is the MP algorithm using a rank 2 orthogonal projection [18]. IAA and ReLIKES perform well as soon as the target lie on their initial grids (which is the case for the strong target when $\mathrm{k}=4$ ). Otherwise (as for $\mathrm{k}=3$ ) they tend to generate some spurious peaks, especially when the target SNR is large. To the best of our knowledge, the setting of a desired false alarm probability remains an open problem with these approaches. On the contrary, the proposed approach performs well, and is able to correctly detect the stronger target and the weaker targets close to them without spurious detections. Of course, it cannot separate the unresolved scatterers but it was not designed for that. It was build to significantly reduces spurious detections.

In Figure 8 and 9, we examine the ability of the considered algorithms to both offer good target detection probability and reduce the probability of detecting a sidelobe (outlier). For that, a single target is considered, made of several unresolved point scatterers, lying on the $\mathrm{k}=4$ grid. The target is detected if a detection occurs inside the resolution cell. If a detection occurs outside the target resolution cell, an outlier is said to be detected. Figure 8 focuses on the chirp radar model (61), where ReLIKES was not implemented due to its computational cost, whereas Figure 9 deals with the common spectral estimation model (44). The results in Figure 8 and 9 show that the proposed method outperforms the others: it offers not only good target detection probability but also a good control of the outlier appearance probability, which is maintained close to the probability of false alarm times the number of independent GLRT tests. OMP, IAA and ReLIKES fail to 


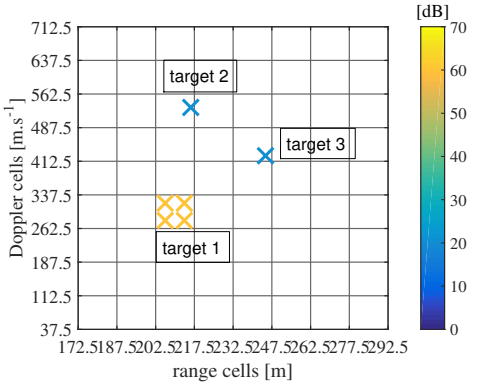

(a) True scene

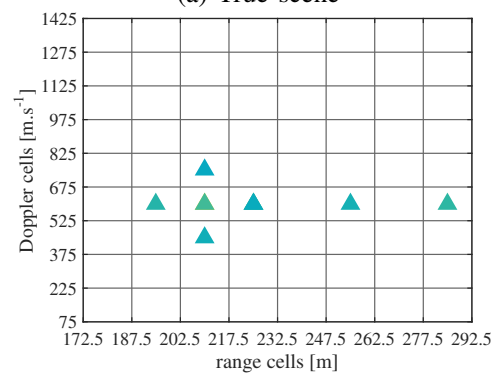

(d) rank 2 projector

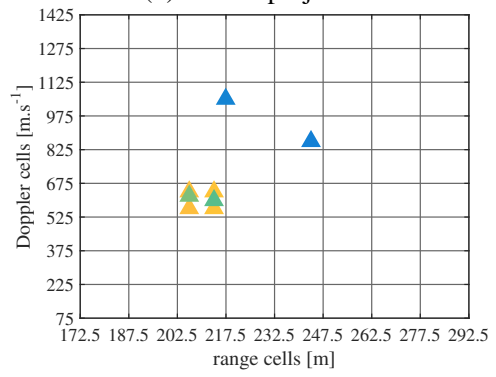

(g) ReLIKES, k=4

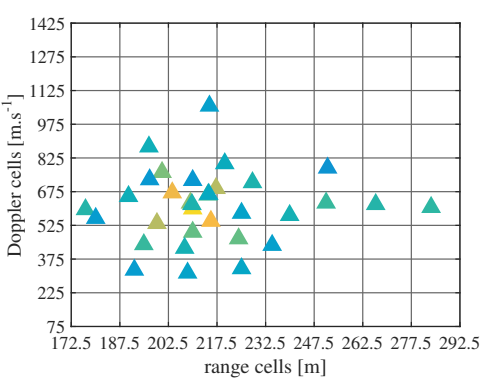

(b) MP off_grid

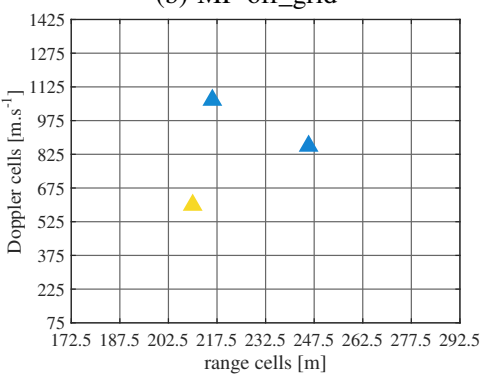

(e) SR MP (proposed)

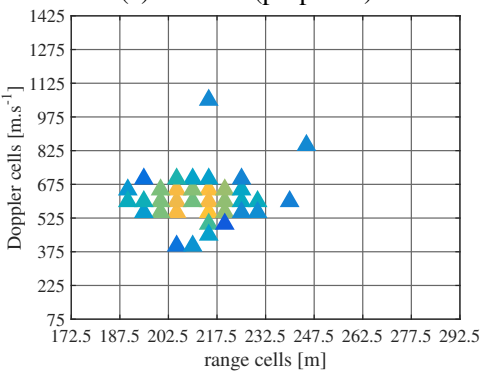

(h) IAA, $\mathrm{k}=3$

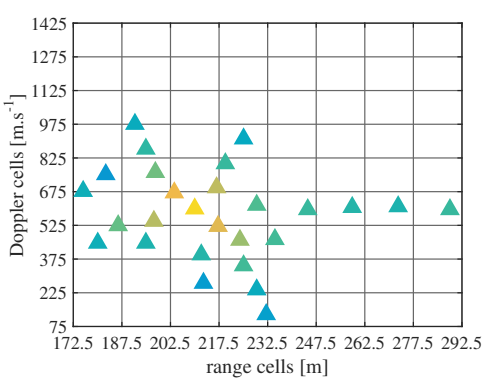

(c) OMP

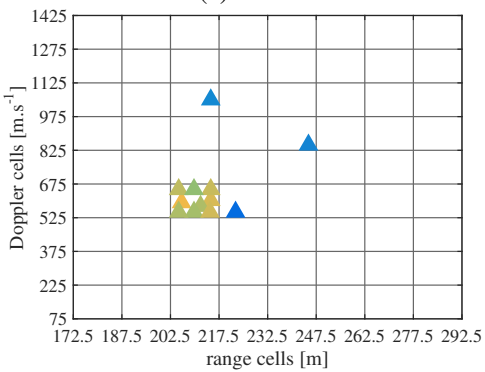

(f) ReLIKES, k=3

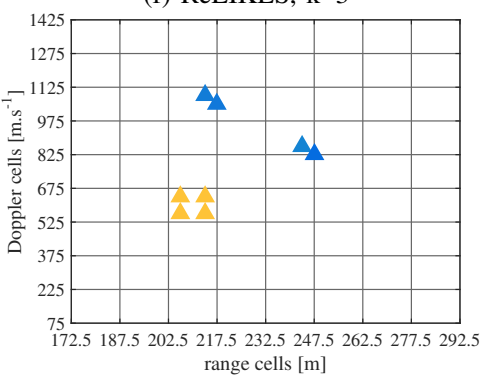

(i) IAA, $\mathrm{k}=4$

Figure 7: Pulsed chirp radar signal case. Output of several algorithms. The unresolved target (target 1) is made of 4 unresolved scatterers, located at $\left(\frac{k}{4} \Delta_{\tau}, \frac{l}{4} \Delta_{f}\right),\{k, l\} \in\{-1,1\} \times\{-1,1\}$, inside their cell, with the same amplitude $\alpha=10^{\frac{S N R_{1}}{20}}$ where $S N R_{1}=60 \mathrm{~dB}$. The two point-spread targets (2 and 3) have the same amplitude $\alpha=10^{\frac{S N R_{2}}{20}}$, where $S N R_{2}=20 \mathrm{~dB}$. $: 20$ $\mathrm{dB}$. Target 2 is on the $\mathrm{k}=3$ grid. Target 3 does not lie on the considered grids. $P=20, B=10 \mathrm{MHz}, T_{s}=\frac{1}{B} \mathrm{~s}, N=50$, $N_{p}=20, \Delta_{\nu}=\frac{1}{T_{p} P}, \Delta_{\tau}=\frac{1}{B}, \lambda=3.10^{-2} \mathrm{~m}, P_{f a}=10^{-6}, \varepsilon=0.3$.

do so over the whole range of SNR under consideration. As observed in Figure 7, IAA and ReLIKES exhibit much better performances when the targets are on the initial grid $(\mathrm{k}=4)$ than when the targets are off the grid $(\mathrm{k}=3)$, where they tend to generate more spurious detections. Since IAA and ReLIKES are iterative algorithms and made of different steps, analyzing their behavior is not trivial. We observe that ReLIKES tends to outperform the IAA, maybe due to the fact that ReLIKES approximates the grid interval by a subspace. The performance breakdown observed in Figure 9 could be due to the fact that at some SNR range the subspace approximation with fixed dimension may not be sufficient to maintain sparsity in the dictionary.

Note that the result of the IAA and ReLIKES is not sparse in itself, one should select the dominant peaks among the estimated amplitudes. Consequently, their performance depend on the peak selection rule. We used the same criterion as in [19] (adding a "noise only" hypothesis), which may not be optimal in any case. One drawback of such an approach is that if several approach are known for model selection, they usually offer only asymptotic guarantees [37] and the low to moderate signal to noise ratio regime for example, is not well known. This may explain the erratic behavior of some curves in Figures 8 and 9.

\section{CONCLUSION}

In this article, we studied the case of unresolved targets, that cause classic matching pursuit (or clean) procedure to produce undesirable detections. We proposed a new method that chooses a projector able to eliminate all contributions in a resolution cell. This projector is thus of rank more than one, contrary to existing procedures. We examined both the choice of the basis and the choice of the rank. The chosen subspace basis, minimizes the residual projection error onto the cleaning region: an explicit solution is found thanks to the SVD of a particular matrix. Interestingly, when the steering vector is composed of linearly spaced phases, this subspace coincides with the well known DPSS vectors. The dimension of the projector is chosen according to the target SNR and the proposed criterion was proved to control the projection 


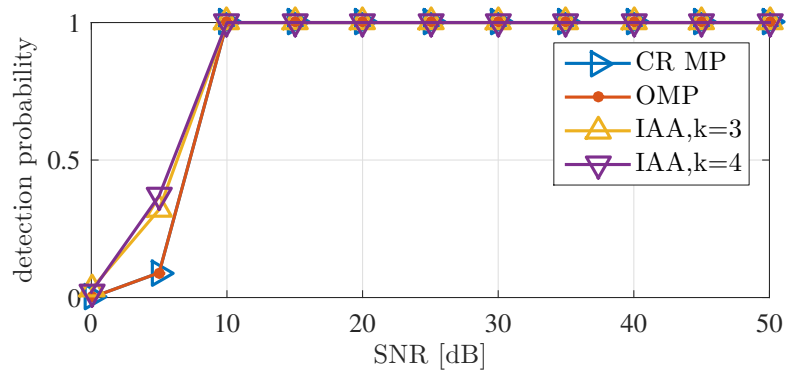

(a) Target detection probability

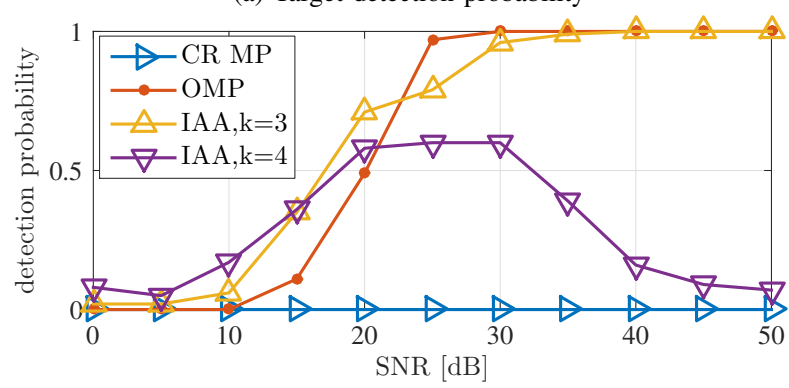

(b) Outlier detection probability

Figure 8: Pulsed chirp radar signal. The single target is made of 4 unresolved scatterers, positioned at $\left(\frac{k}{4} \Delta_{\tau}, \frac{l}{4} \Delta_{f}\right),\{k, l\} \in$ $\{-1,1\}$ with the same amplitude $\alpha=10^{\frac{5 N R}{20}} . N=50, N_{p}=$ $20, P=10, B=10 \mathrm{MHz}, T_{s}=\frac{1}{B} \mathrm{~s}, \Delta_{\nu}=\frac{1}{T_{p} P}, \Delta_{\tau}=\frac{1}{B}$, $\lambda=3.10^{-2} \mathrm{~m}, P_{f a}=10^{-6}, \varepsilon=0.3$, Monte-carlo runs $=100$.

residue so that the maximal sidelobes detection probability after rejection falls close to the desired initial false alarm probability. The proposed technique outperforms existing MP procedures, and other sparse reconstruction methods like IAA and ReLIKES in the sense that it greatly reduces spurious detections caused by unresolved strong targets. The proposed method is not computationally intensive as most information needed for the rejection can be stored off-line. Moreover, as it is based on classic steps already deeply studied in radar, the setting of the parameters (such as the false alarm probability) is much easier and a lot of previous results from the radar literature could be exploited (such as the non-Gaussian environment, for example, or classic CFAR procedures), if desired.

\section{APPENDIX A \\ PROOF OF LEMMA 1}

For $K=1$, we have $\operatorname{Tr}\left[\boldsymbol{\Pi}_{1} \mathbf{B}\right]=\boldsymbol{\pi}_{1}^{H} \mathbf{B} \boldsymbol{\pi}_{1}$, with $\boldsymbol{\pi}_{1}$ of norm 1. It corresponds to a Rayleigh quotient that achieves its maximal value $\lambda_{1}$ for $\boldsymbol{\pi}_{1}=\mathbf{u}_{1}$ [34].

For a given $K$, let us assume that $\operatorname{Tr}\left[\boldsymbol{\Pi}_{K} \mathbf{B}\right]$ is upperbounded by $\sum_{k=1}^{K} \lambda_{k}$ and achieves this maximal value for $\boldsymbol{\pi}_{k}=\mathbf{u}_{k},(1 \leq k \leq K)$. We have straightforwardly

$$
\begin{aligned}
\operatorname{Tr}\left[\boldsymbol{\Pi}_{K+1} \mathbf{B}\right] & =\operatorname{Tr}\left[\boldsymbol{\Pi}_{K} \mathbf{B}\right]+\boldsymbol{\pi}_{K+1}^{H} \mathbf{B} \boldsymbol{\pi}_{K+1} . \\
& \leq \sum_{k=1}^{K} \lambda_{k}+\boldsymbol{\pi}_{K+1}^{H} \mathbf{B} \boldsymbol{\pi}_{K+1}
\end{aligned}
$$

The upper bound in (63) is achieved when $\boldsymbol{\pi}_{k}=\mathbf{u}_{k},(1 \leq$ $k \leq K)$. Then, in this case, since $\pi_{K+1}$ is orthogonal to

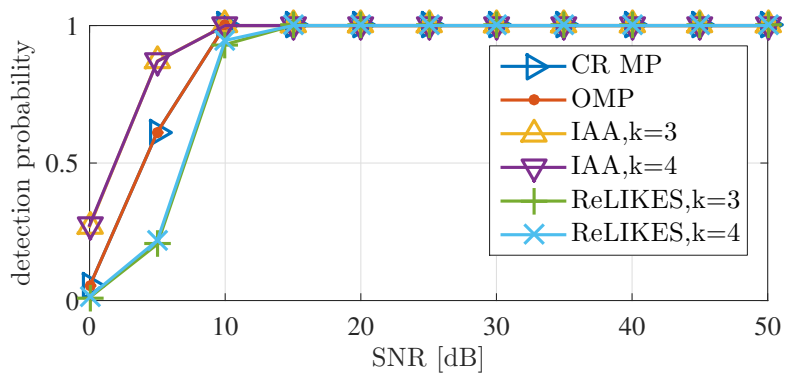

(a) Target detection probability

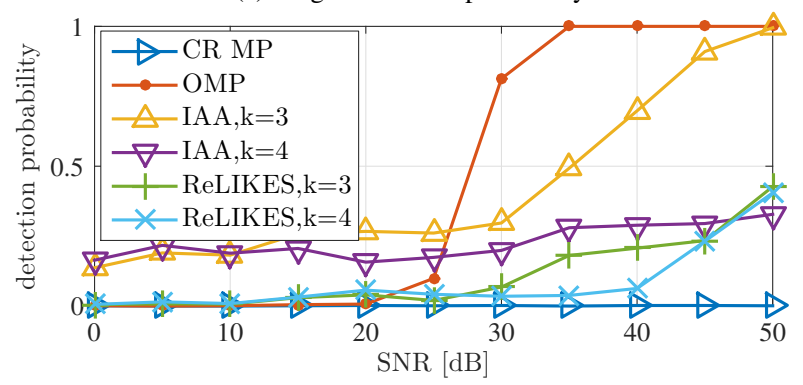

(b) Outlier detection probability

Figure 9: Sinusoids in noise. The signal target is made of two unresolved scatterers located at $f_{1}=\frac{5}{P}$ and $f_{2}=f_{1}+\frac{0.25}{P}$ with the same amplitude $\alpha=10^{\frac{S N R}{20}} . P=20, P_{f a}=10^{-4}$, $\varepsilon=0.3$. Monte carlo runs $=1000$.

$\boldsymbol{\pi}_{k}=\mathbf{u}_{k}(1 \leq k \leq K)$, one finds

$$
\boldsymbol{\pi}_{K+1}^{H} \mathbf{B} \boldsymbol{\pi}_{K+1}=\boldsymbol{\pi}_{K+1}^{H} \mathbf{B}^{\prime} \boldsymbol{\pi}_{K+1},
$$

with $\mathbf{B}^{\prime}=\left[\mathbf{u}_{K+1}, \ldots, \mathbf{u}_{N}\right]^{H} \operatorname{diag}\left(\lambda_{K+1}, \ldots, \lambda_{N}\right)\left[\mathbf{u}_{K+1}, \ldots, \mathbf{u}_{N}\right]$. This new Rayleigh quotient is maximal if and only if $\boldsymbol{\pi}_{K+1}=\mathbf{u}_{K+1}$ and is then equal to $\lambda_{K+1}$. Thus,

$$
\operatorname{Tr}\left[\mathbf{\Pi}_{K+1} \mathbf{B}\right] \leq \sum_{k=1}^{K+1} \lambda_{k},
$$

with equality when $\boldsymbol{\pi}_{k}=\mathbf{u}_{k},(1 \leq k \leq K+1)$. By induction the result is true for any $K \leq N$.

\section{APPENDIX B}

\section{PROOF OF PROPOSITION 4}

Since $\mathbf{U}=\mathbf{U}_{1} \otimes \mathbf{U}_{2}, \Pi_{K_{1}}$ and $\boldsymbol{\Pi}_{K_{2}}$ are formed by the $K_{1}$ and $K_{2}$ first eigenvectors of $\mathbf{U}_{1}$ and $\mathbf{U}_{2}$ respectively, we easily have thanks to the orthogonality of the vectors $\mathbf{v}_{n, i}$, that $\mathbf{U} \boldsymbol{\Pi}_{K}=\boldsymbol{\Pi}_{K} \mathbf{U}$ and then, since $\boldsymbol{\Pi}_{K} \mathbf{U}=\boldsymbol{\Pi}_{K} \mathbf{U} \Pi_{\mathbf{K}}$,

$$
\begin{aligned}
\boldsymbol{\Pi}_{K}^{\perp} \mathbf{U} \Pi_{K}^{\perp} & =\mathbf{U}-\mathbf{U} \boldsymbol{\Pi}_{K} \\
& =\mathbf{U}_{1} \otimes \mathbf{U}_{2}-\left(\mathbf{U}_{1} \boldsymbol{\Pi}_{K_{1}}\right) \otimes\left(\mathbf{U}_{2} \boldsymbol{\Pi}_{K_{2}}\right) .
\end{aligned}
$$

A straightforward calculation gives

$$
\begin{aligned}
\boldsymbol{\Pi}_{K}^{\perp} \mathbf{U} \Pi_{K}^{\perp} & =\sum_{n=1}^{N_{1}} \sum_{n^{\prime}=1}^{N_{2}} \lambda_{\Sigma}\left(n, n^{\prime}\right) \mathbf{v}_{n, 1} \mathbf{v}_{n, 1}^{H} \otimes \mathbf{v}_{n^{\prime}, 2} \mathbf{v}_{n^{\prime}, 2}^{H}, \\
\lambda_{\Sigma}\left(n, n^{\prime}\right) & =\lambda_{n, 1} \lambda_{n^{\prime}, 2}\left(1-H\left(K_{1}-n\right) H\left(K_{2}-n^{\prime}\right)\right),
\end{aligned}
$$

$H$ being the Heaviside function $H(x)=\left\{\begin{array}{ll}0 & \text { if } x<0 \\ 1 & \text { if } x \geq 0\end{array}\right.$. 
The term $1-H\left(K_{1}-n\right) H\left(K_{2}-n^{\prime}\right)$ is equal to 0 , except if $n>K_{1}$ or $n^{\prime}>K_{2}$, then it is equal to 1 .

If $n>K_{1}$, then the maximum of $\lambda_{\Sigma}\left(n, n^{\prime}\right)$ is obviously $\lambda_{K_{1}+1,1} \lambda_{1,2}$. And If $n^{\prime}>K_{2}$, then the maximum of $\lambda_{\Sigma}\left(n, n^{\prime}\right)$ is obviously $\lambda_{1,1} \lambda_{K_{2}+1,2}$. The maximum is clearly the maximum of those two quantities and we have (41).

\section{APPENDIX C}

\section{RANK SELECTION ALGORITHM IN 2D KRONECKER CASE}

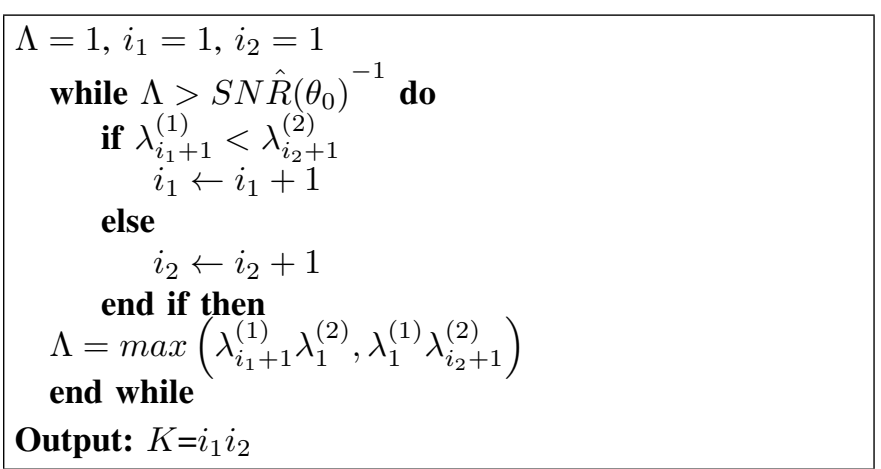

\section{APPENDIX D}

\section{ProOF OF PROPOSITION 3}

Conditionally to $\theta^{\prime}$, the random variable $\left|\left(\alpha \mathbf{u}\left(\theta^{\prime}\right)+\mathbf{n}\right)^{H} \boldsymbol{\Pi}^{\perp} \mathbf{u}(\theta)\right|^{2} / \sigma^{2}\left\|\boldsymbol{\Pi}^{\perp} \mathbf{u}(\theta)\right\|^{2}$ follows a non central $\chi^{2}$ distribution with two degrees of freedom and non centrality parameter

$$
\beta=\frac{\left|\alpha \mathbf{u}^{H}\left(\theta^{\prime}\right) \boldsymbol{\Pi}^{\perp} \mathbf{u}(\theta)\right|^{2}}{\sigma^{2}\left\|\boldsymbol{\Pi}^{\perp} \mathbf{u}(\theta)\right\|^{2}} .
$$

Consequently [38],

$$
P_{s}\left(\theta \mid \theta^{\prime}\right)=Q_{1}\left(\sqrt{2 \beta}, \sqrt{\frac{2 \gamma}{\sigma^{2}\left\|\Pi^{\perp} \mathbf{u}(\theta)\right\|^{2}}}\right),
$$

where

$$
Q_{n}(a, b)=\frac{1}{a^{n-1}} \int_{b}^{+\infty} x^{n} e^{-\frac{x^{2}+a^{2}}{2}} I_{n-1}(a x) d x,
$$

is the generalized Marcum Q function and $I_{n}($.$) is the n$-th order modified Bessel function of first kind.

Applying the Cauchy-Schwarz inequality, we get

$$
\begin{aligned}
\beta & \leq \frac{|\alpha|^{2}}{\sigma^{2}}\left\|\boldsymbol{\Pi}^{\perp} \mathbf{u}\left(\theta^{\prime}\right)\right\|^{2}, \\
& \leq \frac{|\alpha|^{2}}{\sigma^{2}} \mu .
\end{aligned}
$$

We also have $\left\|\boldsymbol{\Pi}^{\perp} \mathbf{u}(\theta)\right\|^{2} \leq 1 . Q_{1}(a, b)$ is increasing with respect to its first argument. Indeed, $\frac{\partial Q_{1}(a, b)}{\partial a}=$ $a\left(Q_{2}(a, b)-Q_{1}(a, b)\right)>0$, since $n \mapsto Q_{n}(a, b)$ is an increasing function [39]. Note also that $b \mapsto Q_{1}(a, b)$ is decreasing. Therefore,

$$
P_{s}\left(\theta \mid \theta^{\prime}\right) \leq Q_{1}\left(\sqrt{\frac{2|\alpha|^{2}}{\sigma^{2}}} \mu, \sqrt{\frac{2 \gamma}{\sigma^{2}}}\right)
$$

and consequently,

$$
\begin{aligned}
\bar{P}_{s}^{\max } & \leq \mathbb{E}_{\theta^{\prime}}\left[Q_{1}\left(\sqrt{\frac{2|\alpha|^{2}}{\sigma^{2}}} \mu, \sqrt{\frac{2 \gamma}{\sigma^{2}}}\right)\right] . \\
& =Q_{1}\left(\sqrt{\frac{2|\alpha|^{2}}{\sigma^{2}}} \mu, \sqrt{\frac{2 \gamma}{\sigma^{2}}}\right) .
\end{aligned}
$$

If $\mu$ is such that $|\alpha|^{2} \mu<\gamma$ then, since [40]

$$
Q_{1}(a, b) \leq e^{-\frac{1}{2}(b-a)^{2}}, \quad b>a \geq 0,
$$

we can write

$$
\bar{P}_{s}^{\max } \leq e^{-\left(\sqrt{\frac{\gamma}{\sigma^{2}}}-\sqrt{\frac{|\alpha|^{2}}{\sigma^{2}} \mu}\right)^{2}} .
$$

In particular, when $\frac{|\alpha|^{2}}{\sigma^{2}} \mu \rightarrow 0$,

$$
e^{-\left(\sqrt{\frac{\gamma}{\sigma^{2}}}-\sqrt{\frac{|\alpha|^{2}}{\sigma^{2}} \mu}\right)^{2}}=e^{-\frac{\gamma}{\sigma^{2}}}\left(1+2 \sqrt{\frac{\gamma}{\sigma^{2}}} \sqrt{\frac{|\alpha|^{2}}{\sigma^{2}} \mu}+o\left(\sqrt{\frac{|\alpha|^{2}}{\sigma^{2}} \mu}\right)\right) \text {, }
$$

and we can deduce (33).

\section{APPENDIX E}

\section{EXTENSION TO MULTIPLE UNRESOLVED SCATTERERS}

Assume that we have $L$ independent scatterers of amplitude $\alpha_{l}(1 \leq l \leq L)$, located in $\theta_{l}^{\prime}(1 \leq l \leq L)$ such that $\theta_{l}^{\prime} \sim \mathcal{U}\left(\mathcal{R}\left(\theta_{0}, \Delta\right)\right)$, and these random variables are statistically independent. Then the received signal can be expressed as:

$$
\mathbf{y}=\sum_{l=1}^{L} \alpha_{l} \mathbf{u}\left(\theta_{l}^{\prime}\right)+\mathbf{n},
$$

and $\mathcal{C}_{K}\left(\theta_{0}, \Delta\right)$ in (14) is now

$$
\mathcal{C}_{\theta_{0}, \Delta}\left(\boldsymbol{\Pi}_{K}\right)=\max _{\theta} \mathbb{E}_{\theta_{1}^{\prime}, \ldots, \theta_{L}^{\prime}}\left[\left|\sum_{l=1}^{L} \alpha_{l} \mathbf{u}^{H}\left(\theta_{l}^{\prime}\right) \boldsymbol{\Pi}_{K}^{\perp} \mathbf{u}(\theta)\right|^{2}\right],
$$

where $\Pi_{K}^{\perp}$ is selected according to (9). With $c_{l}(\theta)=$ $\mathbf{u}^{H}\left(\theta_{l}^{\prime}\right) \boldsymbol{\Pi}_{K}^{\perp} \mathbf{u}(\theta)$

$$
\begin{aligned}
\mathbb{E}_{\theta_{1}^{\prime}, \ldots, \theta_{L}^{\prime}}\left[\left|\sum_{l=1}^{L} \alpha_{l} c_{l^{\prime}}(\theta)\right|^{2}\right]= & \sum_{l, l^{\prime}} \alpha_{l} \alpha_{l^{\prime}}^{*} \mathbb{E}_{\theta_{l}^{\prime}, \theta_{l^{\prime}}^{\prime}}\left[c_{l}(\theta) c_{l^{\prime}}^{*}(\mathbf{8})\right. \\
= & \sum_{l=1}^{L}\left|\alpha_{l}\right|^{2} \mathbb{E}_{\theta_{l}^{\prime}}\left[\left|c_{l}(\theta)\right|^{2}\right] \\
& +\sum_{l \neq l^{\prime}} \alpha_{l} \alpha_{l^{\prime}}^{*}\left|\mathbb{E}_{\theta_{l}^{\prime}}\left[c_{l}(\theta)\right]\right|^{2}(84)
\end{aligned}
$$

since the $\theta_{l}^{\prime}$ are independent and identically distributed,

$$
\begin{aligned}
\mathcal{C}_{\theta_{0}, \Delta}\left(\boldsymbol{\Pi}_{K}\right)= & \max _{\theta}\left(\mathbb{E}_{\theta^{\prime}}\left[\left|\mathbf{u}^{H}\left(\theta^{\prime}\right) \boldsymbol{\Pi}_{K}^{\perp} \mathbf{u}(\theta)\right|^{2}\right] \sum_{l=1}^{L}\left|\alpha_{l}\right|^{2}\right. \\
& \left.+\left|\mathbb{E}_{\theta^{\prime}}\left[\mathbf{u}^{H}\left(\theta^{\prime}\right) \boldsymbol{\Pi}_{K}^{\perp} \mathbf{u}(\theta)\right]\right|^{2} \sum_{l \neq l^{\prime}} \alpha_{l} \alpha_{l^{\prime}}^{*}\right)(85)
\end{aligned}
$$


The first term in the right hand side of (85) is bounded by $\lambda_{K+1} \sum_{l=1}^{L}\left|\alpha_{l}\right|^{2}$. We also have

$$
\begin{aligned}
\left|\mathbb{E}_{\theta^{\prime}}\left[\mathbf{u}^{H}\left(\theta^{\prime}\right) \boldsymbol{\Pi}_{K}^{\perp} \mathbf{u}(\theta)\right]\right|^{2} & \leq \mathbb{E}_{\theta^{\prime}}\left[\left|\mathbf{u}^{H}\left(\theta^{\prime}\right) \boldsymbol{\Pi}_{K}^{\perp} \mathbf{u}(\theta)\right|\right]^{2} \\
& \leq \mathbb{E}_{\theta^{\prime}}\left[\left\|\mathbf{u}^{H}\left(\theta^{\prime}\right) \boldsymbol{\Pi}_{K}^{\perp}\right\|\|\mathbf{u}(\theta)\|\right]^{2} \\
& =\mathbb{E}_{\theta^{\prime}}\left[\left\|\mathbf{u}^{H}\left(\theta^{\prime}\right) \boldsymbol{\Pi}_{K}^{\perp}\right\|\right]^{2} \\
& \leq \mu .
\end{aligned}
$$

Note that $\sum_{l \neq l^{\prime}} \alpha_{l} \alpha_{l^{\prime}}^{*}=2 R e\left(\sum_{l>l^{\prime}} \alpha_{l} \alpha_{l^{\prime}}^{*}\right)$ so that the second term in the right hand side of (85) is real. Thus, if $\sum_{l \neq l^{\prime}} \alpha_{l} \alpha_{l^{\prime}}^{*} \geq 0$ this term is upper-bounded by $\mu \sum_{l \neq l^{\prime}} \alpha_{l} \alpha_{l^{\prime}}^{*}$, and if $\sum_{l \neq l^{\prime}} \alpha_{l} \alpha_{l^{\prime}}^{*}<0$ this term is upper-bounded by zero. Thus, using the Heaviside function $H($.$) again,$

$$
\mathcal{C}_{\theta_{0}, \Delta}\left(\boldsymbol{\Pi}_{K}\right) \leq \sum_{l=1}^{L}\left|\alpha_{l}\right|^{2} \lambda_{K+1}+\mu H\left(\sum_{l \neq l^{\prime}} \alpha_{l} \alpha_{l^{\prime}}^{*}\right) \sum_{l \neq l^{\prime}} \alpha_{l} \alpha_{l^{\prime}}^{*}
$$

Now we shall see how to control $\mathcal{C}_{\theta_{0}, \Delta}\left(\boldsymbol{\Pi}_{K}\right)$ so that a sufficient rejection level is guaranteed. To do so, one should have a look at the SNR matched filter estimate which provides through (19) the rejection level. The averaged MF estimate of the target SNR is

$$
\begin{aligned}
\Lambda & =\mathbb{E}_{\theta_{1}^{\prime}, \theta_{2}^{\prime}, \ldots, \theta_{L}^{\prime}, \mathbf{n}}\left[\left|\mathbf{y}^{H} \mathbf{u}\left(\theta_{0}\right)\right|^{2}\right], \\
& =\sigma^{2}+\mathbb{E}_{\theta_{1}^{\prime}, \theta_{2}^{\prime}, \ldots, \theta_{L}^{\prime}}\left[\left|\sum_{l=1}^{L} \alpha_{l}^{*} \mathbf{u}^{H}\left(\theta_{l}^{\prime}\right) \mathbf{u}\left(\theta_{0}\right)\right|^{2}\right] .
\end{aligned}
$$

Expanding the sum and rearranging terms, we have then

$$
\begin{aligned}
\Lambda= & \sigma^{2}+\sum_{l=1}^{L}\left|\alpha_{l}\right|^{2} \mathbb{E}_{\theta^{\prime}}\left[\left|\mathbf{u}^{H}\left(\theta^{\prime}\right) \mathbf{u}\left(\theta_{0}\right)\right|^{2}\right] \\
& +\sum_{l \neq l^{\prime}} \alpha_{l} \alpha_{l^{\prime}}^{*}\left|\mathbb{E}_{\theta^{\prime}}\left[\mathbf{u}^{H}\left(\theta^{\prime}\right) \mathbf{u}\left(\theta_{0}\right)\right]\right|^{2} .
\end{aligned}
$$

We can upper-bound the second expectation by

$$
\left|\mathbb{E}_{\theta^{\prime}}\left[\mathbf{u}^{H}\left(\theta^{\prime}\right) \mathbf{u}\left(\theta_{0}\right)\right]\right|^{2} \leq \mathbb{E}_{\theta^{\prime}}\left[\left|\mathbf{u}^{H}\left(\theta^{\prime}\right) \mathbf{u}\left(\theta_{0}\right)\right|\right]^{2} \leq 1 \text {. }
$$

As for the first expectation, if $\Delta$ has been chosen according to the main lobe $3 \mathrm{~dB}$ width,

$$
\left|\mathbf{u}^{H}\left(\theta^{\prime}\right) \mathbf{u}\left(\theta_{0}\right)\right|^{2} \geq \frac{1}{2}, \forall \theta^{\prime} \in \mathcal{R}\left(\theta_{0}, \Delta\right),
$$

and consequently,

$$
\frac{1}{2} \leq \mathbb{E}_{\theta^{\prime}}\left[\left|\mathbf{u}^{H}\left(\theta^{\prime}\right) \mathbf{u}\left(\theta_{0}\right)\right|^{2}\right] \leq \lambda_{1} .
$$

We have then

$$
\begin{aligned}
& \sigma^{2}+\frac{1}{2} \sum_{l=1}^{L}\left|\alpha_{l}\right|^{2}+\left[1-H\left(\sum_{l \neq l^{\prime}} \alpha_{l} \alpha_{l^{\prime}}^{*}\right)\right] \sum_{l \neq l^{\prime}} \alpha_{l} \alpha_{l^{\prime}}^{*} \leq \Lambda \\
& \Lambda \leq \sigma^{2}+\lambda_{1}\left(\theta_{0}, \Delta\right) \sum_{l=1}^{L}\left|\alpha_{l}\right|^{2}+H\left(\sum_{l \neq l^{\prime}} \alpha_{l} \alpha_{l^{\prime}}^{*}\right) \sum_{l \neq l^{\prime}} \alpha_{l} \alpha_{l^{\prime}}^{*}
\end{aligned}
$$

where, again, $H($.$) is the Heaviside function. Note that usually$ $\lambda_{1}\left(\theta_{0}, \Delta\right)$ is close to one.
For the considered common steering vectors (44) and (49) we have additionally, with $r\left(\theta^{\prime}-\theta_{0}\right)=\mathbf{u}^{H}\left(\theta^{\prime}\right) \mathbf{u}\left(\theta_{0}\right)$,

$$
\left|r\left(\theta^{\prime}-\theta_{0}\right)\right| \in[\gamma, 1], \forall \theta^{\prime} \in \mathcal{R}\left(\theta_{0}, \Delta\right)
$$

where $\gamma$ can be approximated by $\frac{1}{\sqrt{2}}$. We also have $r(-\theta)=$ $r^{*}(\theta)$, so that

$$
\frac{1}{2} \leq\left|\mathbb{E}_{\theta^{\prime}}\left[\mathbf{u}^{H}\left(\theta^{\prime}\right) \mathbf{u}\left(\theta_{0}\right)\right]\right|^{2} \leq 1 .
$$

We have then

$$
\begin{gathered}
\sigma^{2}+\frac{1}{2} \sum_{l=1}^{L}\left|\alpha_{l}\right|^{2}+\min \left(\frac{1}{2} \sum_{l \neq l^{\prime}} \alpha_{l} \alpha_{l^{\prime}}^{*}, \sum_{l \neq l^{\prime}} \alpha_{l} \alpha_{l^{\prime}}^{*}\right) \leq \Lambda \text { (99) } \\
\Lambda \leq \sigma^{2}+\lambda_{1}\left(\theta_{0}, \Delta\right) \sum_{l=1}^{L}\left|\alpha_{l}\right|^{2}+\max \left(\frac{1}{2} \sum_{l \neq l^{\prime}} \alpha_{l} \alpha_{l^{\prime}}^{*}, \sum_{l \neq l^{\prime}} \alpha_{l} \alpha_{l^{\prime}}^{*}\right),
\end{gathered}
$$

Now, if the phases of the $\alpha_{l}$ are equal then $\sum_{l \neq l^{\prime}} \alpha_{l} \alpha_{l^{\prime}}^{*}=$ $\sum_{l \neq l^{\prime}}\left|\alpha_{l}\right|\left|\alpha_{l^{\prime}}\right| \geq 0$ and (99) and (100) becomes

$$
\begin{gathered}
\sigma^{2}+\frac{1}{2}\left(\sum_{l=1}^{L}\left|\alpha_{l}\right|^{2}+\sum_{l \neq l^{\prime}}\left|\alpha_{l}\right|\left|\alpha_{l^{\prime}}\right|\right) \leq \Lambda, \\
\Lambda \leq \sigma^{2}+\sum_{l=1}^{L}\left|\alpha_{l}\right|^{2} \lambda_{1}\left(\theta_{0}, \Delta\right)+\sum_{l \neq l^{\prime}}\left|\alpha_{l}\right|\left|\alpha_{l^{\prime}}\right| .
\end{gathered}
$$

The term $\sigma^{2}$ in the previous equations can be neglected in high SNR case, which is the main interest here. The mean MF estimate $\left|\mathbf{y}^{H} \mathbf{u}\left(\theta_{0}\right)\right|$ should not be very different (up to $3 \mathrm{~dB}$ ) from $\sum_{l=1}^{L}\left|\alpha_{l}\right|^{2}+\sum_{l \neq l^{\prime}}\left|\alpha_{l}\right|\left|\alpha_{l^{\prime}}\right|=\left|\sum_{l=1}^{L} \alpha_{l}\right|^{2}$. So, choosing $\lambda_{K+1}$ such that $\left|\mathbf{y}^{H} \mathbf{u}(\theta)\right| \lambda_{K+1}\left(\theta_{0}, \Delta\right) \ll \sigma^{2}$, should result approximately in $\lambda_{K+1}\left(\theta_{0}, \Delta\right)\left|\sum_{l=1}^{L} \alpha_{l}\right|^{2} \ll \sigma^{2}$. If we approximate $\lambda_{K+1}\left(\theta_{0}, \Delta\right) \approx \mu$ when $K$ is large enough, we have $\mathcal{C}_{K}\left(\theta_{0}, \Delta\right) \leq\left|\sum_{l=1}^{L} \alpha_{l}\right|^{2} \lambda_{K+1}\left(\theta_{0}, \Delta\right) \ll \sigma^{2}$, and the approach developed for the single scatterer case can be reused.

On the contrary, if the phases are such that $\sum_{l \neq l^{\prime}} \alpha_{l} \alpha_{l^{\prime}}^{*} \leq 0$ then we see that since the mean MF SNR estimate- bounded in (99) and (100) - is susceptible to be inferior to $\sum_{l=1}^{L}\left|\alpha_{l}\right|^{2}$, so that choosing $\lambda_{K+1}$ such that $\left|\mathbf{y}^{H} \mathbf{u}\left(\theta_{0}\right)\right| \lambda_{K+1}\left(\theta_{0}, \Delta\right) \ll \sigma^{2}$ does not provide a tight bound in (96), which can be larger than 1 . So, when the phases of the scatterers are too chaotic, the resulting SNR can be severely underestimated so that the rank of the continuous projector becomes underestimated and the rejection is susceptible to be inefficient.

\section{REFERENCES}

[1] H. L. Van Trees, Optimum Array Processing. John Wiley \& Sons, 2002.

[2] H. Krim and M. Viberg, "Two decades of array signal processing research,” IEEE Signal Processing Magazine, pp. 67-94, 1996.

[3] C. Ren, J. Galy, E. Chaumette, P. Larzabal, and A. Renaux, "High resolution techniques for radar: Myth or reality ?" In proceedings of EUSIPCO 2013, 2013.

[4] S. G. Mallat and Z. Zhang, "Matching pursuits with time-frequency dictionaries," IEEE Transactions on Signal Processing, vol. 41, no. 12, pp. 3397-3415, 1993.

[5] H. L. V. Trees, Detection, Estimation and Modulation Theory, Part III. New York, NY: John Wiley \& Sons, Inc, 2001. 
[6] M. A. Richards, Fundamentals of radar signal processing. Tata McGraw-Hill Education, 2005.

[7] J. Tsao and B. D. Steinberg, "Reduction of sidelobe and speckle artifacts in microwave imaging: the clean technique," IEEE Transactions on Antennas and Propagation, vol. 36, no. 4, pp. 543-556, 1988.

[8] E. J. Candès and M. B. Wakin, "An introduction to compressive sampling," Signal Processing Magazine, IEEE, vol. 25, no. 2, pp. 21-30, 2008.

[9] E. Baransky, G. Itzhak, N. Wagner, I. Shmuel, E. Shoshan, and Y. Eldar, "Sub-nyquist radar prototype: Hardware and algorithm," IEEE Transactions on Aerospace and Electronic Systems, vol. 50, no. 2, pp. 809-822, 2014.

[10] T. Strohmer, "Measure what should be measured: progress and challenges in compressive sensing," IEEE Signal Processing Letters, vol. 19, no. 12, pp. 887-893, 2012.

[11] R. Jagannath, G. Leus, and R. Pribic, "Grid matching for sparse signal recovery in compressive sensing," pp. 111-114, 2012.

[12] A. Fannjiang and W. Liao, "Coherence pattern-guided compressive sensing with unresolved grids," SIAM Journal on Imaging Sciences, vol. 5, no. 1, pp. 179-202, 2012.

[13] T. Huang, Y. Liu, H. Meng, and X. Wang, "Adaptive matching pursuit with constrained total least squares," EURASIP Journal on Advances in Signal Processing, vol. 2012, no. 1, p. 76, 2012.

[14] A. Fannjiang and H.-C. Tseng, "Compressive radar with off-grid targets: a perturbation approach," Inverse Problems, vol. 29, no. 5, 2013.

[15] G. Tang, B. N. Bhaskar, P. Shah, and B. Recht, "Compressed sensing off the grid," IEEE transactions on information theory, vol. 59, no. 11, pp. 7465-7490, 2013.

[16] M. Lasserre, S. Bidon, O. Besson, and F. Le Chevalier, "Bayesian sparse fourier representation of off-grid targets with application to experimental radar data," Signal Processing, pp. 261-273, 2015.

[17] X. Zhang, P. Willett, and Y. Bar-Shalom, "Detection and localization of multiple unresolved extended targets via monopulse radar signal processing," IEEE Transactions on Aerospace and Electronic Systems, vol. 45, no. 2, pp. 455-472, 2009.

[18] M. Cattenoz, L. Savy, and S. Marcos, "Adaptive processing methods for MIMO radar experimental signals," in proceedings of IEEE RADAR 2014, 2014.

[19] T. Yardibi, J. Li, P. Stoica, M. Xue, and A. B. Baggeroer, "Source localization and sensing: A nonparametric iterative approach based on weighted least squares," IEEE Trans. Aerosp. Electron. Syst., vol. 46 , no. 1, pp. 425-443, 2010.

[20] P. Stoica and P. Babu, "Sparse estimation of spectral lines: Grid selection problems and their solutions," IEEE Transactions on Signal Processing, vol. 60, no. 2, pp. 962-967, 2012.

[21] G.-O. Glentis and A. Jakobsson, "Superfast approximative implementation of the IAA spectral estimate," IEEE Transactions on Signal Processing, vol. 60, no. 1, pp. 472-478, 2012.

[22] O. Rabaste and D. Poullin, "Rejection of doppler shifted multipaths in airborne passive radar," IEEE International Radar Conference, pp. $1660-1665,2015$

[23] J. Bosse, O. Rabaste, and D. Poullin, "Matching pursuit via continuous resolution cell rejection in presence of unresolved radar targets," European Signal Processing Conference (EUSIPCO), pp. 1816-1820, 2015.

[24] D. Slepian, "Prolate spheroidal wave functions, fourier analysis, and uncertainty-V: The discrete case," Bell System Technical Journal, vol. 57, no. 5, pp. 1371-1430, 1978.

[25] M. A. Davenport and M. B. Wakin, "Compressive sensing of analog signals using discrete prolate spheroidal sequences," Applied and Computational Harmonic Analysis, vol. 33, no. 3, pp. 438-472, 2012.

[26] M. A. Davenport, S. R. Schnelle, J. Slavinsky, R. G. Baraniuk, M. B Wakin, and P. T. Boufounos, "A wideband compressive radio receiver," Military Communications Conference, MILCOM 2010, pp. 1193-1198, 2010.

[27] C.-Y. Chen and P. P. Vaidyanathan, "MIMO radar space-time adaptive processing using prolate spheroidal wave functions," IEEE Transactions on Signal Processing, vol. 56, no. 2, pp. 623-635, 2008.

[28] P. Forster and G. Vezzosi, "Application of spheroidal sequences to array processing," IEEE International Conference on Acoustics, Speech, and Signal Processing ICASSP'87., vol. 12, pp. 2268-2271, 1987.

[29] Z. Zhu and M. B. Wakin, "Approximating sampled sinusoids and multiband signals using multiband modulated dpss dictionaries," Journal of Fourier Analysis and Applications, pp. 1-48, 2016.

[30] — "Wall clutter mitigation and target detection using discrete prolate spheroidal sequences," 3rd IEEE Int. Workshop on Compressed Sensing
Theory and its Applications to Radar, Sonar and Remote Sensing (CoSeRa), pp. 47-51, 2015

[31] F. Ahmad, J. Qian, and M. G. Amin, "Wall clutter mitigation using discrete prolate spheroidal sequences for sparse reconstruction of indoor stationary scenes," IEEE Transactions on Geoscience and Remote Sensing, vol. 53, no. 3, pp. 1549-1557, 2015.

[32] Z. Zhu and M. B. Wakin, "On the dimensionality of wall and target return subspaces in through-the-wall radar imaging," 4th IEEE International Workshop on Compressed Sensing Theory and its Applications to Radar, Sonar and Remote Sensing (CoSeRa), pp. 110-114, 2016.

[33] T. T. Cai and L. Wang, "Orthogonal matching pursuit for sparse signal recovery with noise," IEEE Transactions on Information theory, vol. 57, no. 7, pp. 4680-4688, 2011.

[34] F. Gantmacher, Matrix Theory. New-York : Chelsea, 1959, vol. I-II.

[35] R. Behrens and L. Scharf, "Signal processing applications of oblique projection operators," IEEE Transactions on Signal Processing, vol. 42, no. 6, pp. 1413-1424, 1994.

[36] J. A. Tropp and A. C. Gilbert, "Signal recovery from random measurements via orthogonal matching pursuit," IEEE Transactions on information theory, vol. 53, no. 12, pp. 4655-4666, 2007.

[37] P. Stoica and Y. Selen, "Model-order selection: a review of information criterion rules," IEEE Signal Processing Magazine, vol. 21, no. 4, pp. 36-47, 2004.

[38] D. A. Shnidman, "The calculation of the probability of detection and the generalized marcum q-function," IEEE Transactions on Information Theory, vol. 35, no. 2, pp. 389-400, 1989.

[39] Y. Sun, Á. Baricz, and S. Zhou, "On the monotonicity, log-concavity, and tight bounds of the generalized Marcum and Nuttall-functions," IEEE Transactions on Information Theory, vol. 56, no. 3, pp. 1166-1186, 2010.

[40] M. K. Simon and M.-S. Alouini, "Exponential-type bounds on the generalized Marcum Q-function with application to error probability analysis over fading channels," IEEE Transactions on Communications, vol. 48 , no. 3 , pp. $359-366,2000$. 